\title{
Spin waves and high-frequency response in layered superconductors with helical magnetic structure
}

\author{
A. E. Koshelev \\ Materials Science Division, Argonne National Laboratory, Argonne, Illinois 60439
}

(Dated: May 26, 2021)

\begin{abstract}
We evaluate the spin-wave spectrum and dynamic susceptibility in a layered superconductor with helical interlayer magnetic structure. We especially focus on the structure in which the moments rotate $90^{\circ}$ from layer to layer realized in the iron pnictide $\mathrm{RbEuFe}_{4} \mathrm{As}_{4}$. While in nonmagnetic superconductors low-frequency magnetic field decays on the distance of the order of the London penetration depth, spin waves mediate its propagation to much larger distances limited by external dissipation mechanisms. The spin-wave spectrum in superconductors is strongly renormalized due to the long-range electromagnetic interactions between the oscillating magnetic moments. This leads to strong enhancement of the frequency of the mode coupled with uniform field and this enhancement exists only within a narrow range of the $c$-axis wave vectors of the order of the inverse London penetration depth. The key feature of materials like $\mathrm{RbEuFe}_{4} \mathrm{As}_{4}$ is that this uniform mode corresponds to the maximum frequency of the spin-wave spectrum with respect to the $c$-axis wave vector. As a consequence, the high-frequency surface resistance acquires a very distinct asymmetric feature spreading between the bare and renormalized frequencies. We also consider excitation of spin waves with the Josephson effect in a tunneling contact between helical-magnetic and conventional superconductors and study the interplay between the spin-wave features and geometrical cavity resonances in the current-voltage characteristics.
\end{abstract}

\section{INTRODUCTION}

Experimental realization, characterization, and understanding of quantum materials have emerged as central topics in modern physics research. Quantum materials have the potential to offer new functionalities enabling novel applications and therefore provide a fundamental basis for future technological advances. Superconductors supporting long-range magnetic order represent a rare class of quantum materials with unique properties caused by the interplay between magnetic and superconducting subsystems 1 4]. As singlet superconductivity and ferromagnetism are strongly incompatible states, the groundstate configurations are always characterized by nonuniform structures of either magnetic moments or superconducting gap parameter. The nature of nonuniform configurations ultimately determines transport and thermodynamic properties of these materials. In the case of strong superconductivity and soft magnetism, it was theoretically predicted that the exchange interaction between two subsystems favors a nonuniform magnetic state either in the form of small-size domains 5 or helical structure 6 for strong and weak magnetic anisotropy, respectively.

Several classes of magnetic singlet superconductors have been discovered and thoroughly characterized. The magnetism in these materials is hosted in the rareearth-element sublattice spatially separated from the conduction-electron sublattice. In spite of high density of rare-earth $(R E)$ local moments, the superconductivity survives because the exchange interaction between two sublattices is relatively weak. Various nonuniform magnetic structures have been revealed in the coexistence regions.

The first two groups of magnetic superconductors discovered half a century ago are ternary molybdenum chalcogenides (Chevrel phases), such as $\mathrm{HoMo}_{6} \mathrm{~S}_{8}$, with superconducting transition at $T_{c} \approx 1.2 \mathrm{~K}[7]$, and ternary rhodium borides, such as $\mathrm{ErRh}_{4} \mathrm{~B}_{4}$, with $T_{c} \approx 8.5 \mathrm{~K}[8$, see detailed reviews 1, 3, 4. In these materials the exchange interaction between magnetic and superconducting subsystems is actually not very weak: the emerging ferromagnetism at sub-Kelvin temperatures destroys superconductivity and causes the reentrance of the normal state. Nevertheless, a narrow coexistence region does exist near the reentrance where an intermediate oscillatory magnetic state is formed [9 11, in qualitative agreement with theoretical expectations.

Another important class of magnetic superconductors is the rare-earth nickel borocarbides $R E \mathrm{Ni}_{2} \mathrm{~B}_{2} \mathrm{C}$, see reviews $12-14$. In contrast to the nearly cubic ternary compounds, these are layered materials composed of magnetic $R E \mathrm{C}$ layers and conducting Ni layers. The superconductivity coexists with different kinds of magnetic order in four compounds with $R E \rightarrow \mathrm{Tm}$, Er, Ho, and Dy. The magnetic moments typically order ferromagnetically within $R E C$ layers and alternate from layer to layer (A-type antiferromagnets). This basic configuration, however, is perturbed in some compounds. Particularly interesting are $\mathrm{Er}$ and Ho compounds where the magnetic transition takes place inside the superconducting state at temperatures comparable with the superconducting transition temperature $(10.5 \mathrm{~K}$ and $8 \mathrm{~K}$, for $\mathrm{Er}$ and Ho, respectively). Magnetic structure in the $\mathrm{ErNi}_{2} \mathrm{~B}_{2} \mathrm{C}$ is characterized by additional in-plane modulation, which is probably caused by interaction with the superconducting sublattice. In addition, a peculiar weak ferromagnetic state appears below $2.3 \mathrm{~K}$, and, contrary to $\mathrm{HoMo}_{6} \mathrm{~S}_{8}$ and $\mathrm{ErRh}_{4} \mathrm{~B}_{4}$, it coexists with superconductivity at lower temperatures. In the Ho compound the magnetic phase diagram is also very rich: the transition 
to the low-temperature A-type antiferromagnetic state occurs via two intermediate incommensurate spiral configurations, one with helix direction along the $c$ axis and another with additional in-plane modulation.

Contrary to singlet Cooper pairing, a rare triplet superconducting state may coexist with uniform ferromagnetism. Such triplet state is realized in uranium-based compounds $\mathrm{UGe}_{2}$, URhGe, and UCoGe, which become superconducting at sub-Kelvin temperature range, inside the ferromagnetic state, see reviews [15 17]. In spite of low transition temperatures, due to triplet pairing, the superconducting state survives up to remarkably high magnetic field, 10-25 teslas. The triplet state is also likely realized in the recently discovered compound $\mathrm{UTe}_{2}$, even though this material is not magnetic [18].

Interest in the physics of magnetic singlet superconductors has been recently reinvigorated by the discovery of the magnetically ordered iron pnictides, in particular, europium-based 122 compounds, see review [19]. The layered structure of these materials is similar to borocarbides: they are composed of the magnetic Eu and conducting FeAs layers. The parent material $\mathrm{EuFe}_{2} \mathrm{As}_{2}$ is a nonsuperconducting compensated multiple-band metal which has the spin-density-wave transition in the FeAs layers at $189 \mathrm{~K}$ and the A-type antiferromagnetic transition in the $\mathrm{Eu}^{2+}$ layers at $19 \mathrm{~K}$ with the magnetic moments aligned along the layers 20 22]. The superconducting state emerges under pressure with the maximum transition temperature reaching $30 \mathrm{~K}$ at $2.6 \mathrm{GPa}$ exceeding the magnetic transition temperature in the Eu sublattice 23]. Superconducting compounds with Eu magnetic order also have been obtained by numerous chemical substitutions on different atomic sites of the parent compound, including isovalent substitutions of $\mathrm{P}$ on the As site [24 29] and of $\mathrm{Ru}$ on the $\mathrm{Fe}$ site [30], electron doping via substitutions of $\mathrm{Co}$ 31] or Ir 32 on the $\mathrm{Fe}$ site, and hole-doping via substitutions of $\mathrm{K} 33$ or $\mathrm{Na} 34$ on the Eu site. The maximum superconducting transition temperature for different substitution series ranges from 22 to $35 \mathrm{~K}$ exceeding the magnetic-transition temperature in Eu layers. Therefore the key feature of these materials is that they have magnetic transition in the $\mathrm{Eu}^{2+}$ sublattice at the temperature scale, comparable with the superconducting transition in FeAs sublattice. The most studied substituted superconductor in this family is $\operatorname{EuFe}\left(\mathrm{As}_{1-x} \mathrm{P}_{x}\right)_{2}$. The superconducting transition temperature reaches a maximum of $26 \mathrm{~K}$ for $x \approx 0.3$ followed by the ferromagnetic transition at $19 \mathrm{~K}$. Contrary to the parent compound, the Eu moments align ferromagnetically along the $c$ axis at $19 \mathrm{~K}[29]$. At lower temperatures, the coexistence of ferromagnetism with superconductivity leads to the formation of the composite domain and vortex-antivortex structure visualized by the decorations [35] and magnetic-force microscopy [36]. This structure has been explained assuming purely electromagnetic coupling between the magnetic moments and superconducting order parameter [37.

A recent addition to the family of Eu-based iron pnic- tides is the stoichiometric 1144 compounds $A \mathrm{EuFe}_{4} \mathrm{As}_{4}$ with $A=\mathrm{Rb}$ 38 42 and $\mathrm{Cs}$ 39, 43] in which every second layer of $\mathrm{Eu}$ in the parent material is replaced with the layer of nonmagnetic $\mathrm{Rb}$ or $\mathrm{Cs}$. These materials have the superconducting transition temperature of $36.5 \mathrm{~K}$, higher than the doped $122 \mathrm{Eu}$ compounds. Such high transition temperature is achieved because of close-tooptimal hole concentration and the absence of disorder caused by random substitutions. On the other hand, the magnetic transition temperature $15 \mathrm{~K}$ is $4 \mathrm{~K}$ lower than in the parent 122 compound, probably due to the weaker interaction between the magnetic layers. These materials are characterized by highly anisotropic easy-axis Eu magnetism [41, 44, 45]. With increasing pressure the superconducting transition temperature decreases and the magnetic transition temperature increases so that at pressures larger that $\sim 7 \mathrm{GPa}$ the superconducting transition already takes place in the magnetically ordered state [46, 47]. Recent resonant X-ray scattering and neutron diffraction measurements demonstrated that the magnetic structure is helical: the $\mathrm{Eu}$ moments align ferromagnetically inside the layers and rotate $90^{\circ}$ from layer to layer [48, 49].

New materials frequently host new physical phenomena. In this paper we investigate spin waves and related properties for layered superconductors with helical magnetic structure with the modulation perpendicular to the layer direction. Spin waves is the most important dynamic characteristic of magnetic materials [5052 and their properties are essential for the emerging spintronics [53, 54] and magnonics [55,57] applications. As the ground-state configuration, the spin-wave spectrum is determined by the exchange and electromagnetic interactions between the moments and by magnetic anisotropy. A key feature of superconducting materials is that the long-wave part of the spin-wave spectrum is renormalized in a nontrivial way by long-range electromagnetic interactions between the oscillating magnetic moments. In the case of a ferromagnetic triplet superconductor with uniform magnetization, the spectrum of spin waves, their excitation by the external electromagnetic waves, and related features in the surface impedance have been considered in Refs. [58, 59]. The spectrum of spin waves in antiferromagnetic singlet superconductors has been evaluated in Ref. 60. Here we extend these considerations to superconductors with helical magnetic structure. While some of our results are valid for a general modulation period, we mostly focus on the case relevant for $\mathrm{RbEuFe}_{4} \mathrm{As}_{4}$, namely, the structure in which the moments rotate $90^{\circ}$ from layer to layer and the easy-plane anisotropy exceeding the interlayer exchange interaction. We evaluate the spin-wave spectrum as a function of the $c$-axis wave vector and find that the mode having a $c$-axis uniform component of the oscillating spins corresponds to the spectrum maximum. This mode is strongly renormalized by the long-range electromagnetic interactions, its frequency increases by the factor of the square root of the magnetic permeability with respect to the bare value 
determined only by local interactions. This enhancement rapidly drops when the $c$-axis wave vector shift exceeds the inverse London penetration depth. This behavior is qualitatively different from the case of ferromagnetic alignment [58, 59], where the frequency of the uniform mode is the smallest frequency of the spectrum. We evaluate the high-frequency surface resistance and find that it acquires a very asymmetric feature with a sharp maximum at the bare uniform-mode frequency and a tail extending up to the renormalized frequency.

We also investigate excitation of spin waves with AC Josephson effect in a tunneling contact between helicalmagnetic and conventional superconductors and study the interplay between spin-wave features and geometrical Fiske resonances in the current-voltage characteristics. This consideration is somewhat related to the excitation of the spin waves by the Josephson effect in the ferromagnetic interlayer in SFS junctions 61, 62. In our case, however, the spin-wave feature in current-voltage characteristic has a very distinct shape due to the unusual spectrum in helical-magnetic superconductor, similar to the feature in the frequency dependence of the surface resistance. Namely, the current is sharply enhanced when the Josephson frequency matches the bare uniform-mode frequency and at higher frequencies this excess current has a long tail extending up to the renormalized frequency.

The paper is organized as follows. In Sec. III we introduce the model and write general relations determining the spin-wave spectrum via the dynamic magnetic susceptibility. In Sec. [II] we consider the helical magnetic ground state. The bare spin-wave spectrum due to the short-range interactions is derived in Sec. IV], In Sec. V], we investigate the response to nonuniform magnetic field and derive the nonlocal dynamic susceptibility. Electromagnetic renormalization of the spectrum is considered in Sec.VI. In Sec. VII, we consider the dynamic equation for smooth magnetization, derive the magnetic boundary condition, and evaluate the frequency dependence of the surface impedance. In Sec. VIII, we investigate the excitation of spin waves by the Josephson effect.

\section{MODEL AND GENERAL EQUATIONS}

We consider a layered magnetic superconductor described by the energy functional

$$
\begin{aligned}
\mathcal{E} & =\mathcal{E}_{m}+\mathcal{E}_{s} \\
& +\int d^{3} \boldsymbol{r}\left(\frac{\boldsymbol{B}^{2}}{8 \pi}-\boldsymbol{B} \boldsymbol{M}+2 \pi \boldsymbol{M}^{2}-\frac{\boldsymbol{H}_{e} \boldsymbol{B}}{4 \pi}\right),
\end{aligned}
$$

where the term

$$
\mathcal{E}_{s}=\int d^{3} \boldsymbol{r} \sum_{i=x, y, z} \frac{1}{8 \pi \lambda_{i}^{2}}\left(A_{i}-\frac{\Phi_{0}}{2 \pi} \nabla_{i} \varphi\right)^{2}
$$

is the kinetic energy of the superconducting subsystem in the London approximation determined by the com- ponents of the penetration depth $\lambda_{i}$ and $\boldsymbol{A}$ is the vector potential determining the local magnetic induction, $\boldsymbol{B}=\boldsymbol{\nabla} \times \boldsymbol{A}$. In the following, we consider the Meissner state and drop the phase of the superconducting order parameter $\varphi$. We assume that the magnetic subsystem is described by the classical quasi-two-dimensional easyplane Heisenberg model

$$
\begin{aligned}
& \mathcal{E}_{m}=-\mathcal{J} \sum_{\langle\boldsymbol{i}, \boldsymbol{j}\rangle, n} \boldsymbol{S}_{\boldsymbol{i}, n} \boldsymbol{S}_{\boldsymbol{j}, n} \\
&+\mathcal{K} \sum_{\boldsymbol{i}, n}\left(2 S_{z, \boldsymbol{i}, n}^{2}-1\right)-\sum_{\boldsymbol{i}, n, \ell>0} \mathcal{J}_{z, \ell} \boldsymbol{S}_{\boldsymbol{i}, n} \boldsymbol{S}_{\boldsymbol{i}, n+\ell},
\end{aligned}
$$

where $\boldsymbol{S}_{\boldsymbol{i}, n}$ is the spin at the site $\boldsymbol{i}$ and in the layer $n$ with the absolute value equal to $S, \mathcal{J}$ is the in-plane exchange constant, $\mathcal{K}$ is the easy-plane anisotropy, and $\mathcal{J}_{z, \ell}$ are the interlayer exchange constants. The exchange constants likely have a substantial RKKY contribution. The behavior of $\mathcal{J}_{z, \ell}$ for $\ell>\xi_{c} / d$ is strongly affected by superconductivity $[6,63$, where $d$ is the separation between the magnetic layers and $\xi_{c}$ is the $c$ axis coherence length. Local spins determine local magnetic moments $\boldsymbol{m}_{\boldsymbol{i}, n}=g \mu_{B} \boldsymbol{S}_{\boldsymbol{i}, n}$ where $\mu_{B}$ is the Bohr magneton. Therefore, the bulk magnetization $\boldsymbol{M}(\boldsymbol{r})$ in Eq. (1) is related to the coarse-grained spin distribution as $\boldsymbol{M}(\boldsymbol{r})=n_{M} g \mu_{B} \boldsymbol{S}(\boldsymbol{r})$, where $n_{M}$ is the bulk density of spins. $\boldsymbol{S}(\boldsymbol{r})$ in this relation is obtained by averaging of $\boldsymbol{S}_{\boldsymbol{i}, n}$ over distances much larger than neighboring spin separations.

Slowly varying in space oscillating magnetization generates macroscopic magnetic fields which couple with this magnetization. This effect is especially important in superconductors where it leads to significant renormalization of the spin-wave spectrum [58, 59]. We will assume that the supercurrent response to the slowly oscillating magnetization can be treated quasistatically. The corresponding equation is obtained by variation of the energy with respect to the vector potential $\boldsymbol{A}$,

$$
\left(\hat{\lambda}^{-2}-\triangle\right) \boldsymbol{A}-4 \pi \boldsymbol{\nabla} \times \boldsymbol{M}=0 .
$$

We can transform this equation into the equation connecting the local magnetic field strength $\boldsymbol{H}=\boldsymbol{B}-4 \pi \boldsymbol{M}$ and magnetization

$$
\boldsymbol{H}+\boldsymbol{\nabla} \times \hat{\lambda}^{2} \nabla \times \boldsymbol{H}=-4 \pi \boldsymbol{M} .
$$

For time-dependent fields, this equation is modified by quasiparticle currents. We neglect this contribution assuming that the time variations are slow. On the other hand, the oscillating magnetic field generates oscillating magnetization due to dynamic magnetic response and the relation between their Fourier components is determined by the dynamic magnetic susceptibility $\hat{\chi}(\boldsymbol{k}, \omega)$,

$$
\boldsymbol{M}(\boldsymbol{k}, \omega)=\hat{\chi}(\boldsymbol{k}, \omega) \boldsymbol{H}(\boldsymbol{k}, \omega) .
$$

Note that the poles of $\hat{\chi}(\boldsymbol{k}, \omega)$ give the bare spin-wave spectrum due to local interactions unrenormalized by 
long-range fields. From Eqs. (5) and (6), we obtain the general linear equation for $\boldsymbol{H}(q, \omega)$ which determines the spectrum of spin waves

$$
\boldsymbol{H}-\boldsymbol{k} \times \hat{\lambda}^{2} \boldsymbol{k} \times \boldsymbol{H}=-4 \pi \hat{\chi}(\boldsymbol{k}, \omega) \boldsymbol{H} .
$$

In the following, we consider a simple geometry of the wave vector oriented along the $z$ direction and isotropic in-plane case, $\lambda_{x}=\lambda_{y} \equiv \lambda$. In this case, Eq. (7) becomes

$$
\left(1+\lambda^{2} k_{z}^{2}\right) H_{\alpha}\left(k_{z}, \omega\right)=-4 \pi \chi_{\alpha \beta}\left(k_{z}, \omega\right) H_{\beta}\left(k_{z}, \omega\right) .
$$

with $\alpha=x, y$. Note that the off-diagonal susceptibility $\chi_{x y}\left(k_{z}, \omega\right)$ is finite in the helical magnetic state. Since $\chi_{y x}\left(k_{z}, \omega\right)=-\chi_{x y}\left(k_{z}, \omega\right)$, we obtain the following equation for the spin-wave spectrum renormalized by longrange electromagnetic interactions

$$
1+\frac{4 \pi\left[\chi_{x x}\left(k_{z}, \omega\right) \pm i \chi_{x y}\left(k_{z}, \omega\right)\right]}{1+\lambda^{2} k_{z}^{2}}=0 .
$$

The dynamic susceptibility $\chi_{\alpha \beta}(\boldsymbol{k}, \omega)$ can be evaluated by solving the Landau-Lifshitz equation

$$
\frac{d \boldsymbol{M}}{d t}=-\gamma\left[\boldsymbol{M} \times \frac{\delta \mathcal{E}_{m}}{\delta \boldsymbol{M}}\right]+\gamma[\boldsymbol{M} \times \boldsymbol{H}]
$$

in the linear order with respect to small deviations of the magnetization from the equilibrium configuration. Here $\gamma=g \mu_{B} / \hbar$ is the gyromagnetic factor. We neglected the damping terms.

\section{MAGNETIC GROUND-STATE CONFIGURATION}

\section{A. Arbitrary modulation wave vector}

We start with consideration of the helical interlayer magnetic ground state determined by the energy in Eq. (3). In the classical description, it is convenient to introduce the unit vectors $\boldsymbol{s}_{\boldsymbol{i}, n}=\left(\cos \phi_{\boldsymbol{i}, n} \cos \theta_{\boldsymbol{i}, n}\right.$, $\left.\sin \phi_{\boldsymbol{i}, n} \cos \theta_{\boldsymbol{i}, n}, \sin \theta_{\boldsymbol{i}, n}\right)$ along the direction of $\boldsymbol{S}_{\boldsymbol{i}, n}$, $\boldsymbol{S}_{\boldsymbol{i}, n}=\boldsymbol{S}_{\boldsymbol{i}, n}$. Then we can rewrite the energy in Eq. (3) as

$$
\begin{aligned}
\mathcal{E}_{m} & =-J \sum_{\langle\boldsymbol{i}, \boldsymbol{j}\rangle, n} \boldsymbol{s}_{\boldsymbol{i}, n} \boldsymbol{s}_{\boldsymbol{j}, n}+K \sum_{\boldsymbol{i}, n}\left(2 s_{z, \boldsymbol{i}, n}^{2}-1\right) \\
& -\sum_{\boldsymbol{i}, n, \ell>0} J_{z, \ell} \boldsymbol{s}_{\boldsymbol{i}, n} \boldsymbol{s}_{\boldsymbol{i}, n+\ell}
\end{aligned}
$$

with new parameters $J=\mathcal{J} S^{2}, K=\mathcal{K} S^{2}$, and $J_{z, \ell}=$ $\mathcal{J}_{z, \ell} S^{2}$. The advantage of the constants $K, J$, and $J_{z, \ell}$ is that they immediately represent the energy scales of the corresponding interactions. Frustrated interlayer interactions may lead to the helical ground state corresponding to $\phi_{\boldsymbol{i}, n}^{(0)}=q n$ and $\theta_{\boldsymbol{i}, n}^{(0)}=0$ 64, 65. The energy per spin for such a state is given by

$$
E_{0}(q)=-J_{z}(q) / 2
$$

where

$$
J_{z}(q)=2 \sum_{\ell=1}^{\infty} J_{z, \ell} \cos (q \ell)
$$

is the discrete Fourier transform of the interlayer interactions.

The total energy also has an electromagnetic (dipole) contribution, which is substantially affected by superconductivity. As follows from Eq. (5), the magnetic field generated by uniformly polarized layers with arbitrary in-plane orientation of magnetization, $\boldsymbol{M}(z)=$ $\sum_{n} \mathcal{M}_{n} \delta\left(z-z_{n}\right)$, is given by

$$
\boldsymbol{H}(z)=-\sum_{n}\left(2 \pi \mathcal{M}_{n} / \lambda\right) \exp \left(-\left|z-z_{n}\right| / \lambda\right)
$$

where $\boldsymbol{M}_{n}=d n_{M} \boldsymbol{m}_{n}$ is the two-dimensional moment densities, $d$ is the separation between the magnetic layers, and $z_{n}=n d$. Note that the superconducting environment leads to the finite magnetic field outside a uniformly polarized layer, contrary to the normal-state case, in which such field is absent. The corresponding magnetic induction and vector-potential are

$$
\begin{aligned}
& \boldsymbol{B}(z)=4 \pi \sum_{n} \boldsymbol{\mathcal { M }}_{n}\left[\delta\left(z-z_{n}\right)-(1 / 2 \lambda) \exp \left(-\left|z-z_{n}\right| / \lambda\right)\right], \\
& \boldsymbol{A}(z)=-2 \pi \sum_{n} \boldsymbol{n}_{z} \times \mathcal{M}_{n} \operatorname{sign}\left(z-z_{n}\right) \exp \left(-\left|z-z_{n}\right| / \lambda\right) .
\end{aligned}
$$

Substituting these distributions into superconducting and magnetic energy terms in Eq. (1), we derive the bulk electromagnetic energy density

$$
\mathcal{F}_{\mathrm{em}}=\frac{\pi}{\lambda L_{z}} \sum_{n, m} \mathcal{M}_{n} \mathcal{M}_{m} \exp \left(-\left|z_{n}-z_{m}\right| / \lambda\right) .
$$

Therefore, for the helical order, $\mathcal{M}_{x, n}=\mathcal{M}_{0} \cos (Q n)$, $\mathcal{M}_{y, n}=\mathcal{M}_{0} \sin (Q n)$, the bulk electromagnetic-energy cost is

$$
\begin{gathered}
\mathcal{F}_{\mathrm{em}}(Q)=\frac{\pi}{\lambda d} \mathcal{M}_{0}^{2} \frac{\sinh (d / \lambda)}{\cosh (d / \lambda)-\cos Q} \\
\approx \frac{2 \pi M_{0}^{2}}{1+2 \lambda^{2}(1-\cos Q) / d^{2}},
\end{gathered}
$$

where $M_{0}=\mathcal{M}_{0} / d=n_{M} m_{0}$ is the bulk saturation magnetization. The corresponding energy per spin $E_{\mathrm{em}}(Q)=$ $\mathcal{F}_{\text {em }}(Q) / n_{M}$ has to be compared with the exchange energy in Eq. (11). In the range $2 \lambda^{2}(1-\cos Q) / d^{2} \gg 1$, this amounts to comparison of the typical dipole energy scale $E_{d 0}=\pi d^{2} n_{M} m_{0}^{2} / \lambda^{2}$ with the interlayer exchange constants $J_{z, \ell}$. Typically the dipole interactions are much weaker than exchange ones. For example, for parameters of $\mathrm{RbEuFe}_{4} \mathrm{As}_{4}, d=1.33 \mathrm{~nm}, n_{M}=5 \cdot 10^{21} \mathrm{~cm}^{-3}$, $m_{0}=7 \mu_{B}$, and $\lambda=100 \mathrm{~nm}$, we estimate $E_{d 0} \approx 10^{-4} \mathrm{~K}$, while the typical magnitude of $J_{z, \ell}$ is $0.1-0.2 \mathrm{~K}$. In the following, we neglect the electromagnetic energy contribution. 
We proceed with evaluation of the ground-state modulation wave vector $Q$. Equation (11) determines the minimum-energy condition at $q=Q$,

$$
\sum_{\ell} \ell J_{z, \ell} \sin (Q \ell)=0
$$

If we keep only three nearest neighbors, this equation becomes

$$
J_{z, 1}+4 J_{z, 2} \cos Q+3 J_{z, 3}\left(4 \cos ^{2} Q-1\right)=0 .
$$

The energy has a minimum at $q=Q$ if

$$
E_{0}^{\prime \prime}(Q)=\sum_{\ell=1}^{\infty} \ell^{2} J_{z, \ell} \cos (Q \ell)>0
$$

The last two equations determine the optimal modulation vector in the case of frustrating interlayer exchange interactions.

\section{B. Case $Q=\pi / 2$}

In the following, we will pay special attention to the case of commensurate modulation with $Q=\pi / 2$ realized in $\mathrm{RbEuFe}_{4} \mathrm{As}_{4}$. In this case, assuming $J_{z, \ell}=0$ for $\ell>3$, the relation in Eq. (17) gives $J_{z, 1}=3 J_{z, 3}$ and

$$
J_{z}(q)=2 J_{z, 1}\left[\cos (q)+\frac{1}{3} \cos (3 q)\right]+2 J_{z, 2} \cos (2 q) .
$$

The condition for the minimum, Eq. (18), simply gives $J_{z, 2}<0$, i.e., the antiferromagnetic next-neighbor interaction. The case $Q=\pi / 2$, however, is special, because within the simplest exchange model, the energy is degenerate with respect to the relative rotation of the two sublattices composed of odd and even layers. Adding interactions with more remote layers does not resolve this issue. The continuous degeneracy is eliminated by the 4-fold crystal anisotropy term, $-K_{4}\left(s_{x, \boldsymbol{i}, n}^{4}+s_{y, \boldsymbol{i}, n}^{4}\right)$. In addition, such anisotropy locks the $Q=\pi / 2$ state within a finite range of the interlayer exchange constants around the relation $J_{z, 1}=3 J_{z, 3}$. Such 4 -fold anisotropy, however, does not completely eliminate the ground-state degeneracy, because the helical state, $\phi_{n}^{(0)}=\pi n / 2$, still has the same energy as the double-periodic state with $\phi_{n}^{(0)}=(0,0, \pi, \pi, 0,0, \ldots)$. The simplest term eliminating the latter degeneracy is the nearest-neighbor biquadratic term $J_{z, b}\left(\boldsymbol{s}_{\boldsymbol{i}, n} \boldsymbol{s}_{\boldsymbol{i}, n+1}\right)^{2}$ with $J_{z, b}>0$. Without the 4-fold anisotropy term, this yields the modified energy

$$
E_{0}(q)=-\sum_{\ell=1}^{\infty} J_{z, \ell} \cos (q \ell)+J_{z, b} \cos ^{2}(q)
$$

and the modified ground-state condition

$$
\sum_{\ell=1}^{\infty} \ell J_{z, \ell} \sin (Q \ell)-2 J_{z, b} \cos (Q) \sin (Q)=0 .
$$

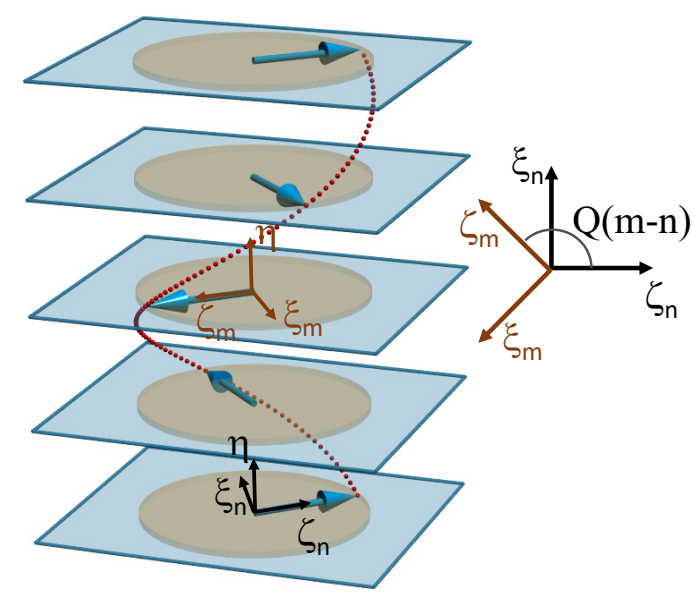

FIG. 1. Local coordinate system $(\varsigma, \xi, \eta)$ used for computation of the spin-wave spectrum.

For three nearest neighbors this gives

$$
J_{z, 1}+4 J_{z, 2} \cos Q+3 J_{z, 3}\left(4 \cos ^{2} Q-1\right)-2 J_{z, b} \cos Q=0 .
$$

For $Q=\pi / 2$ the condition $J_{z, 1}=3 J_{z, 3}$ remains unchanged, while the condition for minimum becomes $2 J_{z, 2}-J_{z, b}<0$. In the following analysis, we will assume the hierarchy of the energy constants $J_{z, b}, K_{4} \ll$ $J_{z, \ell}<K \ll J$. In this case, the degeneracy-breaking terms $\propto J_{z, b}, K_{4}$ select the $Q=\pi / 2$ state but have only a minor impact on the properties discussed in this paper.

\section{BARE SPIN-WAVE SPECTRUM}

\section{A. Arbitrary modulation wave vector}

In this section, we investigate a bare spectrum of spin waves due to the local exchange interactions neglecting coupling to macroscopic fields. We consider spin waves propagating along the direction of helical modulation $(z$ axis) assuming that spin oscillations are uniform in the layer direction. In the following derivations, we drop the in-plane index $\boldsymbol{i}, \boldsymbol{S}_{\boldsymbol{i}, n} \rightarrow \boldsymbol{S}_{n}$. A useful trick allowing for analytical solution is to introduce a local coordinate system $\varsigma, \xi, \eta$ following local equilibrium spin orientation [64. We assume that the $\varsigma$ axis coincides with the equilibrium spin direction at each layer, the $\xi$ axis is perpendicular to this direction in the layer $x y$ plane, and the $\eta$ axis is parallel to the $z$ axis, as illustrated in Fig. 1. Then the $\varsigma, \xi$ axes at the layer $m$ are rotated with respect to those at the layer $n$ by an angle of $Q(m-n)$ corresponding to the coordinate transformation

$$
\begin{aligned}
& \zeta_{m}=\zeta_{n} \cos [Q(m-n)]+\xi_{n} \sin [Q(m-n)], \\
& \xi_{m}=-\zeta_{n} \sin [Q(m-n)]+\xi_{n} \cos [Q(m-n)] .
\end{aligned}
$$


To fix the global coordinate system, we assume $(x, y)=$ $\left(\zeta_{0}, \xi_{0}\right)$ meaning that

$$
\begin{aligned}
& \zeta_{n}=x \cos (Q n)+y \sin (Q n), \\
& \xi_{n}=-x \sin (Q n)+y \cos (Q n) .
\end{aligned}
$$

Correspondingly, the spin components in the rotated and global coordinates are related as

$$
\begin{aligned}
& S_{\zeta n}=S_{x n} \cos (Q n)+S_{y n} \sin (Q n), \\
& S_{\xi n}=-S_{x n} \sin (Q n)+S_{y n} \cos (Q n) .
\end{aligned}
$$

This and inverse transformations can also be presented in the complex form

$$
\begin{aligned}
S_{\zeta n}+i \delta S_{\xi n} & =\left(S_{x n}+i \delta S_{x n}\right) \exp (-i \delta Q n) \\
S_{x n}+i \delta S_{y n} & =\left(S_{\zeta n}+i \delta S_{\xi n}\right) \exp (i \delta Q n)
\end{aligned}
$$

with $\delta= \pm 1$.

The Landau-Lifshitz equation for spin dynamics in the rotated basis can be written as

$$
\begin{gathered}
\dot{S}_{\xi n}=S_{\eta n} h_{\zeta n}-S_{\zeta n} h_{\eta n}, \\
\dot{S}_{\eta n}=S_{\zeta n} h_{\xi n}-S_{\xi n} h_{\zeta n},
\end{gathered}
$$

where $\boldsymbol{h}_{n}=-\partial \mathcal{E}_{m} / \partial \boldsymbol{S}_{n}$ is the local reduced magnetic field acting on spins in the layer $n$, which, according to
Eq. (3), has the components

$$
\begin{aligned}
h_{\zeta n}= & \sum_{m} \mathcal{J}_{z, n-m}\left\{S_{\zeta m} \cos [Q(n-m)]\right. \\
& \left.-S_{\xi m} \sin [Q(n-m)]\right\}, \\
h_{\xi n}= & \sum_{m} \mathcal{J}_{z, n-m}\left\{S_{\zeta m} \sin [Q(n-m)]\right. \\
& \left.+S_{\xi m} \cos [Q(n-m)]\right\}, \\
h_{\eta n}= & \sum_{m} \mathcal{J}_{z, n-m} S_{\eta m}-4 \mathcal{K} S_{\eta n} .
\end{aligned}
$$

For small spin oscillations, the local $\zeta$ component of each spin can be taken as a constant, $S_{\zeta m} \rightarrow S$. Substituting $h_{j n}$ into Eqs. (22a) and (22b), we obtain equations for linear oscillations, $S_{u n}(t)=S_{u n} \exp (i \omega t)$ with $u=\xi, \eta$,

$$
\begin{aligned}
i \omega S_{\xi n} & =S \sum_{m} \mathcal{J}_{z, n-m}\left\{\cos [Q(n-m)] S_{\eta n}-S_{\eta m}\right\} \\
& +4 S \mathcal{K} S_{\eta n}, \\
i \omega S_{\eta n} & =S \sum_{m} \mathcal{J}_{z, n-m} \cos [Q(n-m)]\left(S_{\xi m}-S_{\xi n}\right) .
\end{aligned}
$$

We can see that, in spite of the helical structure, in the rotating coordinates this system is uniform. Fourier transformation $S_{u \mathfrak{q}}=\sum_{n} S_{u n} \exp (-i \mathfrak{q} n)$ yields the $2 \times 2$ linear system

$$
\begin{aligned}
& i \omega S_{\xi \mathfrak{q}}=S\left[\mathcal{J}_{z}(Q)-\mathcal{J}_{z}(\mathfrak{q})+4 \mathcal{K}\right] S_{\eta \mathfrak{q}} \\
& i \omega S_{\eta \mathfrak{q}}=S\left[\frac{\mathcal{J}_{z}(Q+\mathfrak{q})+\mathcal{J}_{z}(Q-\mathfrak{q})}{2}-\mathcal{J}_{z}(Q)\right] S_{\xi \mathfrak{q}}
\end{aligned}
$$

from which we obtain the spectrum

$$
\omega_{s}(\mathfrak{q})=S \sqrt{\left[4 \mathcal{K}+\mathcal{J}_{z}(Q)-\mathcal{J}_{z}(\mathfrak{q})\right]\left[\mathcal{J}_{z}(Q)-\frac{\mathcal{J}_{z}(Q+\mathfrak{q})+\mathcal{J}_{z}(Q-\mathfrak{q})}{2}\right]}
$$

in terms of the reduced wave vector $\mathfrak{q}$. Since $Q$ is the ground-state modulation wave vector, $\mathcal{J}_{z}(q)$ has maximum at $q=Q$, as discussed in Sec. III] This property influences the spectrum shape near $\mathfrak{q}=0$ and $Q$. Spin oscillations in the propagating wave have both in-plane and out-of-plane components. Substituting $\omega_{s}(\mathfrak{q})$ into Eq. 25b), we derive the relation between the spin components in the mode

$$
S_{\eta \mathfrak{q}}=i \sqrt{\frac{\mathcal{J}_{z}(Q)-\frac{\mathcal{J}_{z}(Q+\mathfrak{q})+\mathcal{J}_{z}(Q-\mathfrak{q})}{2}}{4 \mathcal{K}+\mathcal{J}_{z}(Q)-\mathcal{J}_{z}(\mathfrak{q})}} S_{\xi \mathfrak{q}} .
$$

From Eq. 21b, we obtain the in-plane oscillating spin components in real space

$$
\left(\begin{array}{c}
S_{x n} \\
S_{y n}
\end{array}\right)=S_{\xi \mathfrak{q}} \exp (i \mathfrak{q} n)\left(\begin{array}{c}
-\sin (Q n) \\
\cos (Q n)
\end{array}\right) .
$$

We should emphasize that, as $\mathfrak{q}$ represents the wave vector in the rotating-coordinates basis, the real-space spin components $S_{x, y}$ do not behave as $\exp (i \mathfrak{q} n)$. In particular, the mode with $\mathfrak{q}=0$ corresponding to the uniform helix rotation does not generate spin variations uniform in real space.

The mode with $\mathfrak{q}=Q$ will play a key role in the following consideration. For this mode, the in-plane spin components

$$
\left(\begin{array}{c}
S_{x n} \\
S_{y n}
\end{array}\right)=\frac{S_{\xi Q}}{2}\left(\begin{array}{c}
-i+i \exp (2 i Q n) \\
1+\exp (2 i Q n)
\end{array}\right) .
$$

are a superposition of the uniform and $2 Q$ terms. The presence of the uniform $n$-independent component in the $\mathfrak{q}=Q$ mode implies that it can be excited by the oscillating uniform field. The frequency of the mode for 
$\mathfrak{q}=Q$ is given by the geometrical average of the easyplane anisotropy and combination of the interlayer exchange constants,

$$
\omega_{s}(Q)=S \sqrt{2 \mathcal{K}\left[2 \mathcal{J}_{z}(Q)-\mathcal{J}_{z}(2 Q)-\mathcal{J}_{z}(0)\right]}
$$

From Eq. (27), we also obtain the z-axis component of this mode

$$
S_{\eta Q}=i \sqrt{\frac{2 \mathcal{J}_{z}(Q)-\mathcal{J}_{z}(2 Q)-\mathcal{J}_{z}(0)}{8 \mathcal{K}}} S_{\xi Q}
$$

We see that it decreases with increase of the easy-plane anisotropy.

\section{B. Case $Q=\pi / 2$}

In the case $Q=\pi / 2$ and the three-neighbor model discussed in Sec. IIIB, using Eq. (19) and the relation $J_{z}(q)=\mathcal{J}_{z}(q) S^{2}$, we obtain the spectrum

$$
\omega_{s}(\mathfrak{q})=2 S \sqrt{\left\{2 \mathcal{K}-\mathcal{J}_{z, 1}\left[\cos (\mathfrak{q})+\frac{1}{3} \cos (3 \mathfrak{q})\right]+\left|\mathcal{J}_{z, 2}\right|[1+\cos (2 \mathfrak{q})]\right\}\left|\mathcal{J}_{z, 2}\right|[1-\cos (2 \mathfrak{q})]}
$$

This frequency vanishes at $\mathfrak{q}=0$ and $\pi$. The $\mathfrak{q}=0$ mode corresponds to uniform helix rotation. Zero frequency at $\mathfrak{q}=\pi$ is a consequence of the degeneracy with respect to the relative rotation of two sublattices, which is the property of the exchange model in Eq. (3) for $Q=\pi / 2$. These degeneracies are eliminated by the additional terms considered at the end of Sec. III the in-plane 4-fold anisotropy and the nearest-neighbor biquadratic term. The former term generates spin-wave gaps at both $\mathfrak{q}=0$ and $\pi$, while the latter term only generates a gap at $\mathfrak{q}=\pi$. We assume that both these terms are small. We mostly focus on the mode with $\mathfrak{q}=\pi / 2$ which couples with uniform field. This mode is weakly influenced by the additional degeneracy-eliminating terms and we will neglect them below.

Consider the behavior near $\mathfrak{q}=\pi / 2$. Expansion of the frequency in Eq. (32) near this wave vector yields

$$
\omega_{s}\left(\frac{\pi}{2}+q\right) \approx 4 S \sqrt{\mathcal{K}\left|\mathcal{J}_{z, 2}\right|}\left[1-\left(1-\frac{\left|\mathcal{J}_{z, 2}\right|}{\mathcal{K}}\right) \frac{q^{2}}{2}+\frac{\mathcal{J}_{z, 1}}{3 \mathcal{K}} q^{3}\right]
$$

We see that the frequency has a maximum at $\mathfrak{q}=\pi / 2$ for $\mathcal{K}>\left|\mathcal{J}_{z, 2}\right|$. Moreover, one can check that in this case $\omega_{s}(\pi / 2)$ is the largest frequency in the spectrum. We will focus on this case because it is likely realized in $\mathrm{RbEuFe}_{4} \mathrm{As}_{4}$.

\section{Transformation to magnetic unit cell and folded Brillouin zone}

For the modulation vector $\pi / 2$, the magnetic unit cell contains four layers. Correspondingly, the folded magnetic Brillouin zone is four times smaller than the crystalline Brillouin zone. It is therefore useful to present the spin-wave spectrum in the folded Brillouin zone, which better corresponds to a standard crystallographic description. Introducing the index $j$ numbering magnetic unit cells, we present the layer index as $n=4 j+\nu$ with $\nu=1,2,3,4$. Correspondingly, the spins can be repre- sented as

$$
\boldsymbol{S}_{j, \nu}=\boldsymbol{A}_{\nu}(k) \exp (i k j)
$$

where $k$ is the wave vector within the folded Brillouin zone. Using the presentation in Eq. 21b for $S_{\xi n}=$ $S_{0} \exp (i \mathfrak{q} n)$, we write

$$
S_{x, j, \nu}+i \delta S_{y, j, \nu}=i \delta S_{0} \exp \left[i\left(\mathfrak{q}+\delta \frac{\pi}{2}\right)(4 j+\nu)\right],
$$

meaning that $k=4\left(\frac{\pi}{2} m+\mathfrak{q}\right)$ and

$$
A_{x, \nu}^{(m)}(k)+i \delta A_{y, \nu}^{(m)}(k)=i \delta S_{0} \exp \left[i\left(\frac{k}{4}+m \pi+\delta \frac{\pi}{2}\right) \nu\right] .
$$

The integer $m$ should be selected to reduce $k$ to the range $[-\pi, \pi]$. This means that the four modes within such folded Brillouin zone correspond to the frequencies

$$
\begin{aligned}
& \omega_{1}(k)=\omega_{s}(k / 4), \\
& \omega_{2}(k)=\omega_{s}(\pi / 2+k / 4), \\
& \omega_{3}(k)=\omega_{s}(-\pi / 2+k / 4), \\
& \omega_{4}(k)=\omega_{s}(\pi+k / 4),
\end{aligned}
$$

where $\omega_{s}(\mathfrak{q})$ is the spectrum for vector $\mathfrak{q}$ within the original crystalline Brillouin zone, Eq. (32). Note that while $\omega_{1}$ and $\omega_{4}$ are symmetric with respect to $k=0, \omega_{2}$ and $\omega_{3}$ do not have this symmetry and are related as $\omega_{2}(-k)=\omega_{3}(k)$. In addition, the boundary values of the frequencies are connected as $\omega_{1}( \pm \pi)=\omega_{2}(-\pi)=\omega_{3}(\pi)$ and $\omega_{4}( \pm \pi)=\omega_{2}(\pi)=\omega_{3}(-\pi)$.

At the center of the folded Brillouin zone, $k=0$, the first mode corresponds to the uniform spin rotations, $A_{x, \nu}^{(0)}(0)=-S_{0} \sin (\pi \nu / 2), A_{y, \nu}^{(0)}(0)=S_{0} \cos (\pi \nu / 2)$. Its frequency vanishes in the absence of the four-fold anisotropy term. The second and third modes at $k=$ 0 correspond to the modes at $\mathfrak{q}= \pm \pi / 2$ coupled to the uniform field, Eq. $30, \omega_{2}(0)=\omega_{3}(0)=\omega_{s}(\pi / 2)$. The corresponding mode amplitudes are $A_{x, \nu}^{( \pm 1)}(0)=$ $\pm i \frac{(-1)^{\nu}-1}{2} S_{0}, A_{y, \nu}^{( \pm 1)}(0)=\frac{(-1)^{\nu}+1}{2} S_{0}$. The fourth mode at 
$k=0$ corresponds to mutual rotation of odd and even sublattices, $A_{x, \nu}^{(2)}(0)=S_{0} \sin (\pi \nu / 2), A_{y, \nu}^{(2)}(0)=S_{0} \cos (\pi \nu / 2)$, and its frequency also vanishes without degeneracybreaking terms.

\section{RESPONSE TO NONUNIFORM OSCILLATING MAGNETIC FIELD AND DYNAMIC SUSCEPTIBILITY}

\section{A. Arbitrary modulation wave vector}

In this section, we consider the response to the external oscillating nonuniform magnetic field $\tilde{\boldsymbol{h}}_{n}=g \mu_{B} \tilde{\boldsymbol{H}}_{n}$. The real-space components $\left(\tilde{h}_{x n}, \tilde{h}_{y n}, \tilde{h}_{z n}\right)$ correspond to rotating-coordinates components $\left(\tilde{h}_{\xi n}, \tilde{h}_{\zeta n}, \tilde{h}_{\eta n}\right)$ with

$$
\begin{aligned}
& \tilde{h}_{\zeta n}=\tilde{h}_{x n} \cos (Q n)+\tilde{h}_{y n} \sin (Q n), \\
& \tilde{h}_{\xi n}=-\tilde{h}_{x n} \sin (Q n)+\tilde{h}_{y n} \cos (Q n),
\end{aligned}
$$

and $\tilde{h}_{\eta n}=\tilde{h}_{z n}$. In the presence of such external field, equations for the linear spin oscillations, Eqs. 25, become

$$
\begin{aligned}
& i \omega S_{\xi \mathfrak{q}}-S\left[\mathcal{J}_{z}(Q)-\mathcal{J}_{z}(\mathfrak{q})+4 \mathcal{K}\right] S_{\eta \mathfrak{q}}=-S \tilde{h}_{\eta \mathfrak{q}}, \\
& i \omega S_{\eta \mathfrak{q}}+S\left[\mathcal{J}_{z}(Q)-\frac{\mathcal{J}_{z}(Q+\mathfrak{q})+\mathcal{J}_{z}(Q-\mathfrak{q})}{2}\right] S_{\xi \mathfrak{q}}=S \tilde{h}_{\xi \mathfrak{q}}
\end{aligned}
$$

where $\tilde{h}_{\alpha \mathfrak{q}}$ is the Fourier transform of $\tilde{h}_{\alpha n}$. The solution of these equations can be presented as

$$
\begin{aligned}
S_{\xi \mathfrak{q}} & =\chi_{\xi \xi}^{S}(\mathfrak{q}, \omega) \tilde{h}_{\xi \mathfrak{q}}+\chi_{\xi \eta}^{S}(\mathfrak{q}, \omega) \tilde{h}_{\eta \mathfrak{q}}, \\
S_{\eta \mathfrak{q}} & =\chi_{\eta \xi}^{S}(\mathfrak{q}, \omega) \tilde{h}_{\xi \mathfrak{q}}+\chi_{\eta \eta}^{S}(\mathfrak{q}, \omega) \tilde{h}_{\eta \mathfrak{q}},
\end{aligned}
$$

where we defined the susceptibility components in the rotating-coordinates basis,

$$
\begin{aligned}
\chi_{\xi \xi}^{S}(\mathfrak{q}, \omega) & =-\frac{S^{2}\left[\mathcal{J}_{z}(Q)-\mathcal{J}_{z}(\mathfrak{q})+4 \mathcal{K}\right]}{\omega^{2}-\omega_{s}^{2}(\mathfrak{q})}, \\
\chi_{\eta \eta}^{S}(\mathfrak{q}, \omega) & =-\frac{S^{2}\left[\mathcal{J}_{z}(Q)-\frac{\mathcal{J}_{z}(Q+\mathfrak{q})+\mathcal{J}_{z}(Q-\mathfrak{q})}{2}\right]}{\omega^{2}-\omega_{s}^{2}(\mathfrak{q})}, \\
\chi_{\xi \eta}^{S}(\mathfrak{q}, \omega) & =\chi_{\eta \xi}^{S *}(\mathfrak{q}, \omega)=\frac{i \omega S}{\omega^{2}-\omega_{s}^{2}(\mathfrak{q})},
\end{aligned}
$$

and $\omega_{s}(\mathfrak{q})$ is given by Eq. 26.

Equations (38) give the susceptibility components in the helically-rotating coordinates. To study interactions with macroscopic fields, however, we need the susceptibility in real space. As follows from Eq. 28, the spin Fourier components in real coordinates are given by

$$
\begin{aligned}
S_{x q} & =-\frac{S_{\xi, q+Q}-S_{\xi, q-Q}}{2 i}, \\
S_{y q} & =\frac{S_{\xi, q+Q}+S_{\xi, q-Q}}{2} .
\end{aligned}
$$

We emphasize that here and below the wave vector $q$ corresponding to real space is distinguished from the 'fractur' wave vector $\mathfrak{q}$ in the rotated basis which we used above. We use the result for $S_{\xi \mathfrak{q}}$ from Eq. 37a, in which we substitute the field Fourier components

$$
\tilde{h}_{\xi q}=\frac{1}{2} \sum_{\delta= \pm 1}\left[i \delta \tilde{h}_{x, q+\delta Q}+\tilde{h}_{y, q+\delta Q}\right]
$$

following from Eq. $35 \mathrm{~b}$. This yields the spin response in real coordinates

$$
\begin{aligned}
& S_{x q}=\chi_{x x}^{S}(q, \omega) \tilde{h}_{x, q}+\chi_{x y}^{S}(q, \omega) \tilde{h}_{y q} \\
& +\sum_{\delta= \pm 1}\left\{\frac{\chi_{\xi \xi}^{S}(q+\delta Q, \omega)}{4}\left[\tilde{h}_{x, q+2 \delta Q}+i \delta \tilde{h}_{y, q+2 \delta Q}\right]\right. \\
& \left.\quad-\frac{\delta \chi_{\xi \eta}^{S}(q+\delta Q, \omega)}{2 i} \tilde{h}_{z, q+\delta Q}\right\} \\
& S_{y q}=\chi_{y x}^{S}(q, \omega) \tilde{h}_{x, q}+\chi_{y y}^{S}(q, \omega) \tilde{h}_{y q} \\
& +\sum_{\delta= \pm 1}\left\{\frac{\chi_{\xi \xi}^{S}(q+\delta Q, \omega)}{4}\left[i \delta \tilde{h}_{x, q+2 \delta Q}+\tilde{h}_{y, q+2 \delta Q}\right]\right. \\
& \left.+\frac{\chi_{\xi \eta}^{S}(q+\delta Q, \omega)}{2} \tilde{h}_{z, q+\delta Q}\right\}
\end{aligned}
$$

with the real-space spin susceptibility components

$\chi_{x x}^{S}(q, \omega)=\chi_{y y}^{S}(q, \omega)=\frac{\chi_{\xi \xi}^{S}(Q+q, \omega)+\chi_{\xi \xi}^{S}(Q-q, \omega)}{4}$,

$\chi_{x y}^{S}(q, \omega)=-\chi_{y x}^{S}(q, \omega)=-\frac{\chi_{\xi \xi}^{S}(Q+q, \omega)-\chi_{\xi \xi}^{S}(Q-q, \omega)}{4 i}$.

As expected, in addition to the usual diagonal response at the same wave vector, the helical magnetic structure also generates nondiagonal susceptibility and responses at the wave vectors shifted by the modulation wave vector $Q$. Note that the bulk magnetic susceptibility $\chi_{\alpha \beta}\left(k_{z}, \omega\right)$ in Eq. (6) is related to the dynamic spin susceptibility as 1

$$
\chi_{\alpha \beta}\left(k_{z}, \omega\right)=n_{M}\left(g \mu_{B}\right)^{2} \chi_{\alpha \beta}^{S}\left(d k_{z}, \omega\right) .
$$

We will be mostly interested in the smooth spin response to smooth field with the wave vectors much smaller than $Q$. In this case, we can drop the shortwave length terms $\tilde{h}_{x}(q \pm m Q)$ with $m \neq 0$, i.e., keep only the first lines in Eqs. (39a) and (39b). In addition, to obtain the long-wave length response, we use the small- $q$ expansion (recall that $\mathcal{J}_{z}^{\prime \prime}(Q)<0$ ),

$$
\chi_{x x}^{S}(q, \omega) \approx \frac{1}{4} \sum_{\delta= \pm 1} \frac{-S^{2}\left[\frac{\left|\mathcal{J}_{z}^{\prime \prime}(Q)\right|}{2} q^{2}+4 \mathcal{K}\right]}{\omega^{2}-\omega_{s}^{2}(Q)+\delta a_{s} q+c_{s} q^{2}}
$$

\footnotetext{
1 In our notations, $k_{z}$ and $q$ in Eq. 40a are the dimensional and dimensionless $c$-axis wave vectors, respectively, with $q=d k_{z}$
} 
with $a_{s}=2 S^{2} \mathcal{K} \mathcal{J}_{z}^{\prime}(2 Q)$ and

$$
\begin{aligned}
c_{s} & =S^{2}\left\{\mathcal{K}\left[\mathcal{J}_{z}^{\prime \prime}(0)+\mathcal{J}_{z}^{\prime \prime}(2 Q)\right] .\right. \\
& \left.-\frac{\left|\mathcal{J}_{z}^{\prime \prime}(Q)\right|}{2}\left[\mathcal{J}_{z}(Q)-\frac{\mathcal{J}_{z}(0)+\mathcal{J}_{z}(2 Q)}{2}\right]\right\} .
\end{aligned}
$$

Note that for incommensurate-modulation wave vector $Q$, the linear coefficient $a_{s}$ is finite meaning that the frequency $\omega_{s}(q)$ does not have an extremum at $q=Q$. The important particular cases of this result include the response to the uniform oscillating field

$$
\chi_{x x}^{S}(0, \omega)=-\frac{2 S^{2} \mathcal{K}}{\omega^{2}-2 S^{2} \mathcal{K}\left[2 \mathcal{J}_{z}(Q)-\mathcal{J}_{z}(0)-\mathcal{J}_{z}(2 Q)\right]},
$$

which features the antiferromagnetic resonance at the uniform mode frequency, $\omega=\omega_{s}(Q)$, Eq. (30), and the static uniform susceptibility

$$
\chi_{x x}^{S}(0,0) \approx \frac{1}{2 \mathcal{J}_{z}(Q)-\mathcal{J}_{z}(0)-\mathcal{J}_{z}(2 Q)},
$$

which only depends on the interlayer exchange constants. The long-range off-diagonal component $\chi_{x y}^{S}(q, \omega)$ is given by

$$
\chi_{x y}^{S}(q, \omega) \approx-\frac{i}{2} \frac{S^{2}\left[\frac{\left|\mathcal{J}_{z}^{\prime \prime}(Q)\right|}{2} q^{2}+4 \mathcal{K}\right] a_{s} q}{\left[\omega^{2}-\omega_{s}^{2}(Q)+c_{s} q^{2}\right]^{2}-a_{s}^{2} q^{2}} .
$$

It vanishes for $q \rightarrow 0$ proportionally to $q$,

$$
\chi_{x y}^{S}(q, \omega) \approx \frac{-2 i S^{2} \mathcal{K} a_{s} q}{\left\{\omega^{2}-2 S^{2} \mathcal{K}\left[2 \mathcal{J}_{z}(Q)-\mathcal{J}_{z}(0)-\mathcal{J}_{z}(2 Q)\right]\right\}^{2}},
$$

meaning that the transverse spin response is proportional to the field gradient $S_{x} \propto \partial \tilde{h}_{y} / \partial z$.

\section{B. Case $Q=\pi / 2$}

For the commensurate modulation with $Q=\pi / 2$, the spin response, Eq. (39a), simplifies as

$$
\begin{gathered}
S_{x q}=\chi_{x x}^{S}(q, \omega)\left[\tilde{h}_{x, q}+\tilde{h}_{x, \pi-q}\right]+\chi_{x y}^{S}(q, \omega)\left[\tilde{h}_{y q}+\tilde{h}_{y, \pi-q}\right] \\
-\sum_{\delta= \pm 1} \frac{\delta}{2 i} \chi_{\xi \eta}^{S}\left(q+\delta \frac{\pi}{2}, \omega\right) \tilde{h}_{z, q+\delta \frac{\pi}{2}},
\end{gathered}
$$

where the diagonal susceptibility, Eq, 40a, explicitly is given by

$$
\chi_{x x}^{S}(q, \omega)=\frac{1}{4} \sum_{\delta= \pm 1} \frac{-S^{2}\left[\mathcal{J}_{z}(\pi / 2)-\mathcal{J}_{z}(\delta q+\pi / 2)+4 \mathcal{K}\right]}{\omega^{2}-S^{2}\left[\mathcal{J}_{z}\left(\frac{\pi}{2}\right)-\mathcal{J}_{z}\left(\delta q+\frac{\pi}{2}\right)+4 \mathcal{K}\right]\left[\mathcal{J}_{z}\left(\frac{\pi}{2}\right)-\frac{\mathcal{J}_{z}(q)+\mathcal{J}_{z}(\pi-q)}{2}\right]},
$$

and we used the relation $\chi_{x x}^{S}(\pi-q)=\chi_{x x}^{S}(q)$.

In the small- $q$ expansion, Eq. (42), the linear term in the denominator $\propto a_{s}$ vanishes, since $\mathcal{J}_{z}^{\prime}(2 Q) \equiv \mathcal{J}_{z}^{\prime}(\pi)=$ 0 , and the quadratic-term coefficient becomes

$$
\begin{aligned}
& c_{s}=S^{2}\left[\mathcal{K}\left(\mathcal{J}_{z}^{\prime \prime}(0)+\mathcal{J}_{z}^{\prime \prime}(\pi)\right) .\right. \\
& \left.-\frac{\left|\mathcal{J}_{z}^{\prime \prime}(\pi / 2)\right|}{2}\left(\mathcal{J}_{z}(\pi / 2)-\frac{\mathcal{J}_{z}(0)+\mathcal{J}_{z}(\pi)}{2}\right)\right] .
\end{aligned}
$$

For the three-neighbor model, Eq. 19, this coefficient acquires a simple form,

$$
c_{s}=16 S^{2}\left|\mathcal{J}_{z, 2}\right|\left(\mathcal{K}-\left|\mathcal{J}_{z, 2}\right|\right) .
$$

The behavior of the off-diagonal component is very different from the case of incommensurate modulation. It vanishes in the static case and for finite frequency in the small- $q$ limit it behaves as

$$
\begin{aligned}
\chi_{x y}^{S}(q, \omega) & \approx \frac{i S^{2} \mathcal{J}_{z}^{\prime \prime \prime}(\pi / 2) \omega^{2} q^{3}}{12\left(\omega^{2}-\omega_{s}^{2}\left(\frac{\pi}{2}\right)\right)^{2}} \\
& \approx-\frac{4 i S^{2} \mathcal{J}_{z, 1} \omega^{2} q^{3}}{3\left(\omega^{2}-16 S^{2} \mathcal{K}\left|\mathcal{J}_{z, 2}\right|\right)^{2}},
\end{aligned}
$$

i.e., it vanishes $\propto q^{3}$ for $q \rightarrow 0$. This behavior allows us to neglect the off-diagonal component in the further phenomenological considerations.

\section{ELECTROMAGNETIC RENORMALIZATION OF SPECTRUM IN SUPERCONDUCTING STATE}

\section{A. Arbitrary modulation vector}

In this section, we consider the renormalization of the spin-wave spectrum in the superconducting state due to the long-range electromagnetic interactions between the local moments using Eq. (8) in terms of the reduced wave 

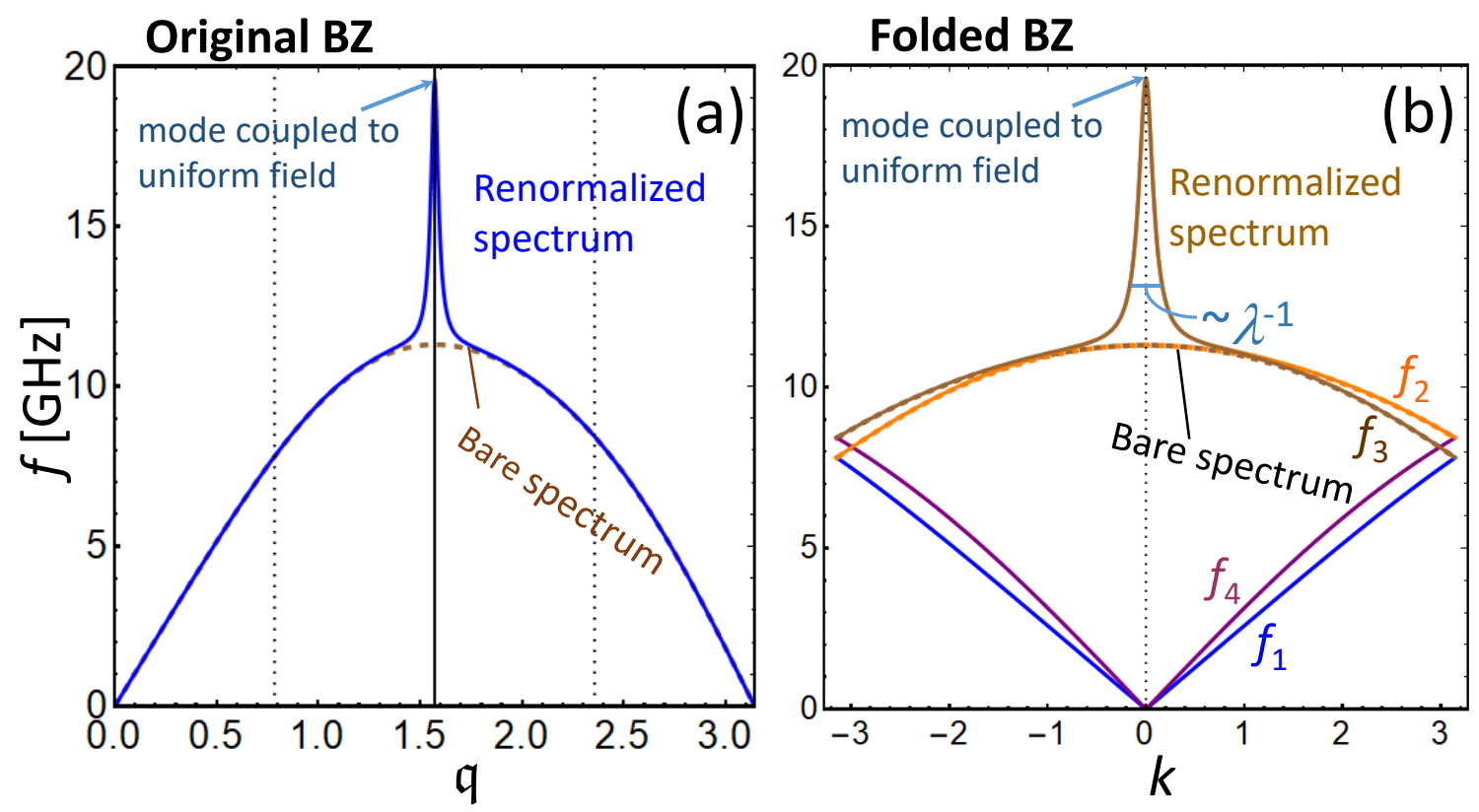

FIG. 2. The representative spectrum of spin waves for the helical structure with $Q=\pi / 2$ in (a) original and (b) folded Brillouin zones for the typical parameters of $\mathrm{RbEuFe}_{4} \mathrm{As}_{4}$. The dashed lines in both plots show the bare spectra obtained without taking into account the renormalization caused by the coupling to macroscopic magnetic field.

vector $q=d k_{z}$. Using Eq. (41) connecting the spin and magnetic susceptibilities and the relation

$$
\begin{aligned}
\chi_{x x}^{S}(q) \pm i \chi_{x y}^{S}(q) & =\frac{1}{2} \chi_{\xi \xi}^{S}(Q \mp q) \\
= & -\frac{1}{2} \frac{S^{2}\left[\mathcal{J}_{z}(Q)-\mathcal{J}_{z}(Q \mp q)+4 \mathcal{K}\right]}{\omega^{2}-\omega_{s}^{2}(Q \mp q)}
\end{aligned}
$$

following from Eqs. 40a) and 40b, we obtain the equation

$$
1+(\lambda / d)^{2} q^{2}-2 \pi \frac{n_{M} m_{0}^{2}\left[\mathcal{J}_{z}(Q)-\mathcal{J}_{z}(Q \mp q)+4 \mathcal{K}\right]}{\omega^{2}-\omega_{s}^{2}(Q \mp q)}=0
$$

for the renormalized spin-wave spectrum, $\omega=\Omega_{s}(Q \mp q)$. The solution of this equation is

$$
\begin{aligned}
\Omega_{s}^{2}(Q+q) & =\omega_{s}^{2}(Q+q) \\
+ & \frac{2 \pi n_{M} m_{0}^{2}\left[\mathcal{J}_{z}(Q)-\mathcal{J}_{z}(Q+q)+4 \mathcal{K}\right]}{1+(\lambda / d)^{2} q^{2}} .
\end{aligned}
$$

The second term gives the spin-wave frequency enhancement due to the long-range electromagnetic interactions. The maximum enhancement is realized near $q=0$ corresponding to the uniform mode, Eqs. (29) and (30). Using the presentation for the static magnetic susceptibility $\chi_{x x}(0,0)=2 n_{M} m_{0}^{2} \mathcal{K} / \omega_{s}^{2}(Q)$ following from Eqs. 41, (44), and (30), we can rewrite Eq. (52) for $q=0$ as $\Omega_{s}^{2}(Q)=\left[1+4 \pi \chi_{x x}(0,0)\right] \omega_{s}^{2}(Q)$ meaning that the renormalized frequency of the uniform mode is

$$
\Omega_{s}(Q)=\sqrt{\mu_{x 0}} \omega_{s}(Q)
$$

where $\mu_{x 0}=1+4 \pi \chi_{x x}(0,0)$ is the static magnetic permeability. Neglecting a weak $q$ dependence in the nominator of the second term in Eq. (52), we can rewrite the frequency renormalization for $q \ll 1$ in a somewhat more transparent form as $\Omega_{s}^{2}(Q+q) \approx \omega_{s}^{2}(Q+q)+$ $4 \pi \chi_{x x}(0,0) \omega_{s}^{2}(Q) /\left[1+(\lambda / d)^{2} q^{2}\right]$.

\section{B. Case $Q=\pi / 2$}

The key features of the commensurate state with $Q=$ $\pi / 2$ are that (i)the frequency $\omega_{s}(\mathfrak{q})$ has maximum at $\mathfrak{q}=Q$ (for $\mathcal{K}>\left|\mathcal{J}_{z, 2}\right|$ ) and (ii)the off-diagonal spin susceptibility vanishes as $q^{3}$ for $q \rightarrow 0$ and therefore its contribution in Eq. (8) can be neglected. Based on the results of Sec. VB, we can represent the dynamics magnetic susceptibility as

$$
\chi_{x x}\left(k_{z}, \omega\right) \approx-\frac{\chi_{x x}(0,0) \omega_{s}^{2}(Q)}{\omega^{2}-\omega_{s}^{2}(Q)+c_{s} d^{2} k_{z}^{2}}
$$

with the static susceptibility $\chi_{x x}(0,0)=$ $n_{M}\left(g \mu_{B}\right)^{2} /\left(8\left|\mathcal{J}_{z, 2}\right|\right)$ and $c_{s}$ is given by Eqs. (49) and (50). The key difference from the ferromagnetic state [58, 59] is the opposite sign of the quadratic coefficient, since in our case the spin-wave frequency has a maximum at $k_{z}=0$ (corresponding to $\mathfrak{q}=\pi / 2$ ).

Solution of Eq. (8) gives the renormalized spectrum in the vicinity of $\mathfrak{q}=Q$ in terms of the reduced wave vector $q=d k_{z}$,

$$
\Omega_{s}^{2}(Q+q)=\omega_{s}^{2}(Q)\left[1+\frac{4 \pi \chi_{x x}(0,0)}{1+(\lambda / d)^{2} q^{2}}\right]-c_{s} q^{2} .
$$


In particular, the renormalization of the uniform mode is again given by Eq. (53). In the folded Brillouin zone discussed in Sec. IV B 1 this mode corresponds to second and third modes at $k=0$, Eqs. (34b) and (34c).

Figure 2 shows spectrum of spin waves in both the original and folded Brillouin zone for the parameters typical for $\mathrm{RbEuFe}_{4} \mathrm{As}_{4}$. Namely, we took $S=7 / 2$, $\mathcal{J}_{z, 1}=0.05 \mathrm{~K}, \mathcal{J}_{z, 2}=-0.01 \mathrm{~K}, \mathcal{K}=0.15 \mathrm{~K}, \lambda=70 \mathrm{~nm}$, and $\mu_{x 0}=3$. For these parameters the bare maximum frequency is $\sim 11 \mathrm{GHz}$. This frequency is strongly enhanced in the superconducting state due to electromagnetic renormalization. This renormalization rapidly decreases for $(\lambda / d)|q-\pi / 2|, 4(\lambda / d) k>1$. We deliberately took a somewhat large value of $\mathcal{J}_{z, 1}$ to enhance the difference between $f_{2}(k)$ and $f_{3}(k)$. For a more realistic choice $\mathcal{J}_{z, 1} \lesssim\left|\mathcal{J}_{z, 2}\right|$ these frequencies become indistinguishable.

\section{DYNAMIC EQUATION FOR SMOOTH MAGNETIZATION, MAGNETIC BOUNDARY CONDITION, AND SURFACE IMPEDANCE}

In this section we consider magnetization response to the alternating magnetic field at the surface and derive the magnetic boundary condition. Here and below, we limit ourselves to the commensurate case $Q=\pi / 2$, for which the frequency of the $z$-axis uniform mode is maximal. As follows from the shape of the susceptibility, Eq. (54), a phenomenological equation for the in-plane magnetization in the case of uniform in-plane field is

$$
\chi_{0}^{-1}\left(1+\omega_{0}^{-2} \frac{\partial^{2}}{\partial t^{2}}+\zeta_{0}^{2} \nabla_{z}^{2}\right) \boldsymbol{M}=\boldsymbol{H}
$$

with $\chi_{0}=\chi_{x x}(0,0), \omega_{0}^{2}=\omega_{s}^{2}(Q)$, and $\zeta_{0}^{2}=c_{s} d^{2} / \omega_{s}^{2}(Q)$. This equation is only valid for smoothly varying magnetization, i. e. for $\zeta_{0}\left|\nabla_{z} M\right| \ll 1$. On the other hand, the local magnetic field $\boldsymbol{H}$ is connected with the magnetization as

$$
\left(1-\lambda^{2} \nabla_{z}^{2}\right) \boldsymbol{H} \approx-4 \pi \boldsymbol{M}
$$

The magnetic length scale $\zeta_{0}$ is much smaller than the London penetration depth $\lambda$. We find the magnetization response to the external oscillating magnetic field. This corresponds to the boundary condition for $\boldsymbol{H}(z, t)$ at the surface, $z=0$,

$$
\boldsymbol{H}(0, t)=\boldsymbol{H}_{0} \exp (i \omega t)
$$

This condition has to be supplemented by the boundary condition for the magnetization, which is usually assumed as

$$
\nabla_{z} M(0, t)=0
$$

We look for the oscillating magnetization and field at the semispace $z>0$ in the form

$$
\begin{aligned}
\boldsymbol{M}(z, t) & =\sum_{\alpha} \boldsymbol{M}_{0 \alpha} \exp \left(i \omega t-\kappa_{\alpha}(\omega) z\right) \\
\boldsymbol{H}(z, t) & =\sum_{\alpha} \boldsymbol{H}_{0 \alpha} \exp \left(i \omega t-\kappa_{\alpha}(\omega) z\right) .
\end{aligned}
$$

In the absence of internal dissipation mechanisms, the parameters $\kappa_{\alpha}(\omega)$ may be either purely real or purely imaginary. It is clear that in the former case $\kappa_{\alpha}(\omega)$ has to be positive. Care should taken to select the correct sign for purely imaginary $\kappa_{\alpha}(\omega)$. Since for the spectrum described by Eq. (56) the group velocity has the opposite sign with respect to the wave vector $q=\operatorname{Im}\left[\kappa_{\alpha}(\omega)\right]$, the energy flows away from the surface for negative $\operatorname{Im}\left[\kappa_{\alpha}(\omega)\right]$. Substituting the above distributions into Eqs. (56) and (57), we obtain equations connecting the vectors $\boldsymbol{M}_{0 \alpha}$ and $\boldsymbol{H}_{0 \alpha}$

$$
\begin{aligned}
\left(1-\lambda^{2} \kappa_{\alpha}^{2}\right) \boldsymbol{H}_{0 \alpha} & \approx-4 \pi \boldsymbol{M}_{0 \alpha} \\
\left(1-\omega^{2} / \omega_{0}^{2}+\zeta_{0}^{2} \kappa_{\alpha}^{2}\right) \boldsymbol{M}_{0 \alpha} & =\chi_{0} \boldsymbol{H}_{0 \alpha}
\end{aligned}
$$

which give the quadratic equation for $\kappa_{\alpha}^{2}(\omega)$

$$
\left(1-\omega^{2} / \omega_{0}^{2}+\zeta_{0}^{2} \kappa_{\alpha}^{2}\right)\left(1-\lambda^{2} \kappa_{\alpha}^{2}\right)+\mu_{x 0}-1=0
$$

Solution of this equation is

$$
\begin{aligned}
\kappa_{\alpha}^{2} & =\frac{\lambda^{-2}+\left(\omega^{2} / \omega_{0}^{2}-1\right) \zeta_{0}^{-2}}{2}+\delta_{\alpha} \sqrt{\frac{\left[\lambda^{-2}+\left(\omega^{2} / \omega_{0}^{2}-1\right) \zeta_{0}^{-2}\right]^{2}}{4}+\zeta_{0}^{-2} \lambda^{-2}\left(\mu_{x 0}-\omega^{2} / \omega_{0}^{2}\right)} \\
& =\frac{\lambda^{-2}+\left(\omega^{2} / \omega_{0}^{2}-1\right) \zeta_{0}^{-2}}{2}+\delta_{\alpha} \sqrt{\frac{\left[\lambda^{-2}-\left(\omega^{2} / \omega_{0}^{2}-1\right) \zeta_{0}^{-2}\right]^{2}}{4}+\zeta_{0}^{-2} \lambda^{-2}\left(\mu_{x 0}-1\right) .}
\end{aligned}
$$

We select $\delta_{1}=\operatorname{sign}\left[\lambda^{-2}+\left(\omega^{2} / \omega_{0}^{2}-1\right) \zeta_{0}^{-2}\right]$ and $\delta_{2}=-\delta_{1}$. Such choice implies that $\left|\kappa_{1}(\omega)\right|>\left|\kappa_{2}(\omega)\right|$ in the whole frequency range. Note that this solution is only formally valid in the frequency range where $\zeta_{0}\left|\kappa_{1}(\omega)\right| \ll 1$ corresponding to the validity range of Eq. (56). In particular, the result for $\kappa_{1}(\omega)$ is not valid for the static case at 
$\omega=0$.

Consider important special cases of Eq. (62). At the bare uniform frequency, $\omega=\omega_{0}$, we obtain

$$
\begin{aligned}
\kappa_{\alpha}^{2}\left(\omega_{0}\right) & =\frac{\lambda^{-2}}{2} \pm \frac{\lambda^{-1}}{2} \sqrt{\lambda^{-2}+4 \zeta_{0}^{-2}\left(\mu_{x 0}-1\right)} \\
& \approx \pm \zeta_{0}^{-1} \lambda^{-1} \sqrt{\mu_{x 0}-1}
\end{aligned}
$$

while at renormalized frequency $\omega=\sqrt{\mu_{x 0}} \omega_{0}$, we have

$$
\begin{aligned}
& \kappa_{1}^{2}\left(\sqrt{\mu_{x 0}} \omega_{0}\right)=\lambda^{-2}+\left(\mu_{x 0}-1\right) \zeta_{0}^{-2}, \\
& \kappa_{2}^{2}\left(\sqrt{\mu_{x 0}} \omega_{0}\right)=0 .
\end{aligned}
$$

However, in the latter case the value of $\kappa_{1}$ is already beyond the applicability range of Eq. (56). Since $\zeta_{0} \ll \lambda$, the inequality $\lambda^{-1} \ll\left|\omega^{2} / \omega_{0}^{2}-1\right| \zeta_{0}^{-1}$ is satisfied almost everywhere, except a narrow region where the frequency is very close to $\omega_{0}$. Away from this region, we can expand $\kappa_{\alpha}^{2}(\omega)$ with respect to $\left(\omega^{2} / \omega_{0}^{2}-1\right)^{-1} \zeta_{0} / \lambda$, which yields

$$
\begin{aligned}
& \kappa_{1}^{2} \approx\left(\omega^{2} / \omega_{0}^{2}-1\right) \zeta_{0}^{-2}+\frac{\left(\mu_{x 0}-1\right) \lambda^{-2}}{\omega^{2} / \omega_{0}^{2}-1} \\
& \kappa_{2}^{2} \approx \lambda^{-2} \frac{\omega^{2} / \omega_{0}^{2}-\mu_{x 0}}{\omega^{2} / \omega_{0}^{2}-1}
\end{aligned}
$$

meaning that the parameters $\kappa_{1}$ and $\kappa_{2}$ mostly describe magnetic and superconducting decay, respectively. The approximation is valid until the second term in $\kappa_{1}^{2}$ is small with respect to the first one giving a somewhat more accurate condition for the expansion $\left|\omega^{2} / \omega_{0}^{2}-1\right| \gg$ $\sqrt{\mu_{x 0}-1} \zeta_{0} / \lambda$. In addition, the condition $\zeta_{0}\left|\kappa_{1}\right| \ll 1$ implies that the result for $\kappa_{1}$ is only valid for $\left|\omega^{2} / \omega_{0}^{2}-1\right| \ll$ 1. However, the result for $\kappa_{2}^{2}$ in Eq. 64b corresponds to the approximation of local magnetic response, $\zeta_{0} \rightarrow 0$, and it remains valid even when the condition $\zeta_{0}\left|\kappa_{1}\right| \ll 1$ breaks, e.g., in the limit $\omega \rightarrow 0$. In the immediate vicinity of the frequency $\omega_{0}$, in the range $\left|\omega^{2} / \omega_{0}^{2}-1\right| \ll$ $\sqrt{\mu_{x 0}-1} \zeta_{0} / \lambda$, the parameters $\kappa_{\alpha}^{2}$ can be evaluated as

$$
\begin{aligned}
\kappa_{\alpha}^{2} & \approx \pm \zeta_{0}^{-1} \lambda^{-1} \sqrt{\mu_{x 0}-1}+\frac{\lambda^{-2}+\left(\omega^{2} / \omega_{0}^{2}-1\right) \zeta_{0}^{-2}}{2} \\
& \pm \frac{\left[\lambda^{-2}-\left(\omega^{2} / \omega_{0}^{2}-1\right) \zeta_{0}^{-2}\right]^{2}}{8 \zeta_{0}^{-1} \lambda^{-1} \sqrt{\mu_{x 0}-1}}
\end{aligned}
$$

This region is characterized by a very strong mixing of spin and supercurrent oscillations. The key observation is that, in contrast to nonmagnetic superconductors, where low-frequency magnetic field decays on the distance of the order of the London penetration depth, in our case for frequency smaller than $\sqrt{\mu_{x 0}} \omega_{0}$ one of the parameters $\kappa_{\alpha}$ is complex meaning that the oscillating magnetic field penetrates at much larger distance limited by external dissipation mechanisms.

We now proceed with evaluation of the vector coefficients $\boldsymbol{M}_{0 \alpha}$ and $\boldsymbol{H}_{0 \alpha}$ from Eqs. (61) using the boundary conditions in Eqs. (58) and (59). Substituting the

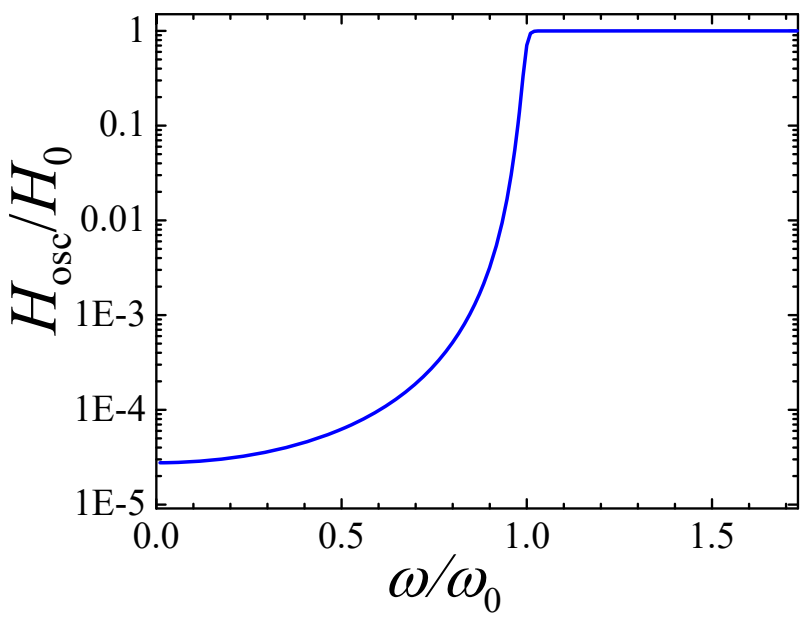

FIG. 3. The frequency dependence of the amplitude of the oscillating field $H_{\text {osc }}$ determining the long-range penetration of the microwave field mediated by the spin waves. We assumed $\zeta_{0}=0.02 \lambda$ and $\mu_{x 0}=3$. The plot terminates at $\omega / \omega_{0}=\sqrt{\mu_{x 0}}$, where $H_{\mathrm{osc}}$ abruptly vanishes.

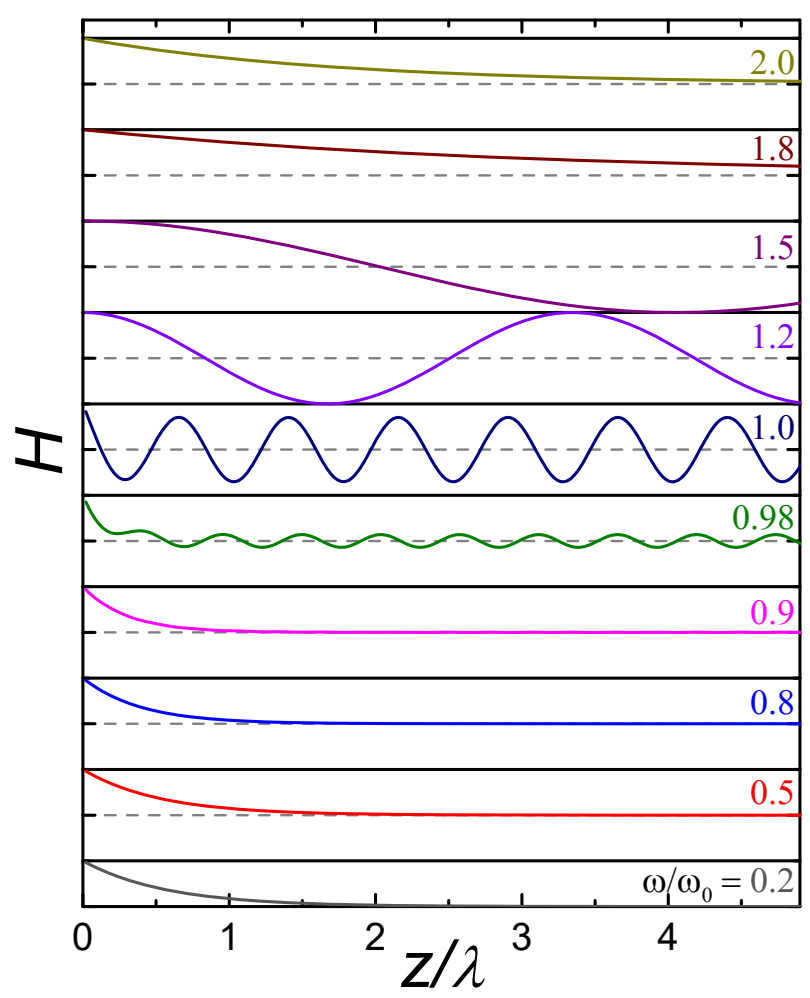

FIG. 4. Series of the coordinate profiles of the microwave magnetic field inside superconductor for different frequencies. A key feature is a pronounced propagating wave in the range $1 \lesssim \omega / \omega_{0}<\sqrt{\mu_{x 0}} \approx 1.73$. Such propagating wave is also present for $\omega / \omega_{0}<1$, but, due to a very small amplitude, it is invisible for the used vertical scale. 
relation $\boldsymbol{H}_{0 \alpha} \approx-\frac{1}{1-\lambda^{2} \kappa_{\alpha}^{2}} 4 \pi \boldsymbol{M}_{0 \alpha}$ into the boundary condition for $\boldsymbol{H}$, we obtain a $2 \times 2$ linear system for the magnetization coefficients

$$
\begin{aligned}
\kappa_{1} \boldsymbol{M}_{01}+\kappa_{2} \boldsymbol{M}_{02} & =0 \\
-\frac{1}{1-\lambda^{2} \kappa_{1}^{2}} \boldsymbol{M}_{01}-\frac{1}{1-\lambda^{2} \kappa_{2}^{2}} \boldsymbol{M}_{02} & =\frac{\boldsymbol{H}_{0}}{4 \pi},
\end{aligned}
$$

which yields the solution

$$
\left(\begin{array}{l}
\boldsymbol{M}_{01} \\
\boldsymbol{M}_{02}
\end{array}\right)=\frac{\left(-\lambda^{2} \kappa_{1}^{2}\right)\left(1-\lambda^{2} \kappa_{2}^{2}\right) \boldsymbol{H}_{0} / 4 \pi}{\left(\kappa_{1}-\kappa_{2}\right)\left[1-\lambda^{2}\left(\kappa_{1}^{2}+\kappa_{1} \kappa_{2}+\kappa_{2}^{2}\right)\right]}\left(\begin{array}{c}
\kappa_{2} \\
-\kappa_{1}
\end{array}\right) .
$$

The corresponding field components are

$$
\begin{aligned}
\left(\begin{array}{c}
\boldsymbol{H}_{01} \\
\boldsymbol{H}_{02}
\end{array}\right) & =\frac{\boldsymbol{H}_{0}}{\left(\kappa_{1}-\kappa_{2}\right)\left[1-\lambda^{2}\left(\kappa_{1}^{2}+\kappa_{1} \kappa_{2}+\kappa_{2}^{2}\right)\right]} . \\
& \times\left(\begin{array}{c}
-\kappa_{2}\left(1-\lambda^{2} \kappa_{2}^{2}\right) \\
\kappa_{1}\left(1-\lambda^{2} \kappa_{1}^{2}\right)
\end{array}\right) .
\end{aligned}
$$

The amplitude of the field inside the superconductor with oscillating coordinate dependence, $\boldsymbol{H}_{\mathrm{osc}}$, corresponding to purely imaginary $\kappa_{\alpha}$ is given by $\boldsymbol{H}_{01}$ for $\omega<\omega_{0}$ and by $\boldsymbol{H}_{02}$ for $\omega_{0}<\omega<\sqrt{\mu_{x 0}} \omega_{0}$. It determines the long-propagating microwave field mediated by the spin waves. Figure 3 shows the frequency dependence of the ratio $H_{\mathrm{osc}} / H_{0}$. We can see that the oscillatory component rapidly increases when the frequency approaches $\omega_{0}$ from below and becomes very close to unity within the range $\omega_{0}<\omega<\sqrt{\mu_{x 0}} \omega_{0}$. It abruptly vanishes at $\omega=\sqrt{\mu_{x 0}} \omega_{0}$. Figure 4 illustrates the coordinate profiles of the microwave magnetic field inside superconductor, $H(z)=\operatorname{Re}\left[H_{1} \exp \left(-\kappa_{1} z\right)+H_{2} \exp \left(-\kappa_{2} z\right)\right]$, for different frequencies. We see that the pronounced oscillating contribution emerges near $\omega \sim \omega_{0}$ and dominates in the range $\omega_{0}<\omega<\sqrt{\mu_{x 0}} \omega_{0} \approx 1.73 \omega_{0}$, while the corresponding wave length of oscillations increases as the frequency approaches $\sqrt{\mu_{x 0}} \omega_{0}$. Slightly above this frequency, the microwave field monotonically decreases but with very large decay length.

The interaction between the magnetic superconductor and outside world can be conveniently formulated in terms the boundary condition connecting the gradient $\nabla_{z} \boldsymbol{H}$ with the field at the surface. From Eq. (67), we obtain

$$
\begin{aligned}
\left.\nabla_{z} \boldsymbol{H}\right|_{z}=0 & =-\kappa_{1} \boldsymbol{H}_{01}-\kappa_{2} \boldsymbol{H}_{02} \\
& =-\frac{\kappa_{1} \kappa_{2}\left(\kappa_{1}+\kappa_{2}\right)}{\kappa_{1}^{2}+\kappa_{1} \kappa_{2}+\kappa_{2}^{2}-\lambda^{-2}} \boldsymbol{H}_{0}
\end{aligned}
$$

Using the relations $\kappa_{1} \kappa_{2}=-i \zeta_{0}^{-1} \lambda^{-1} \sqrt{\mu_{x 0}-\omega^{2} / \omega_{0}^{2}}$ and $\kappa_{1}^{2}+\kappa_{2}^{2}-\lambda^{-2}=\left(\omega^{2} / \omega_{0}^{2}-1\right) \zeta_{0}^{-2}$, we can rewrite this boundary condition as

$$
\nabla_{z} \boldsymbol{H}=-\eta_{\omega} \boldsymbol{H} / \lambda
$$

with

$$
\eta_{\omega}=\frac{-i \zeta_{0} \sqrt{\mu_{x 0}-\omega^{2} / \omega_{0}^{2}}\left(\kappa_{1}+\kappa_{2}\right)}{\omega^{2} / \omega_{0}^{2}-1-i \zeta_{0} / \lambda \sqrt{\mu_{x 0}-\omega^{2} / \omega_{0}^{2}}}
$$

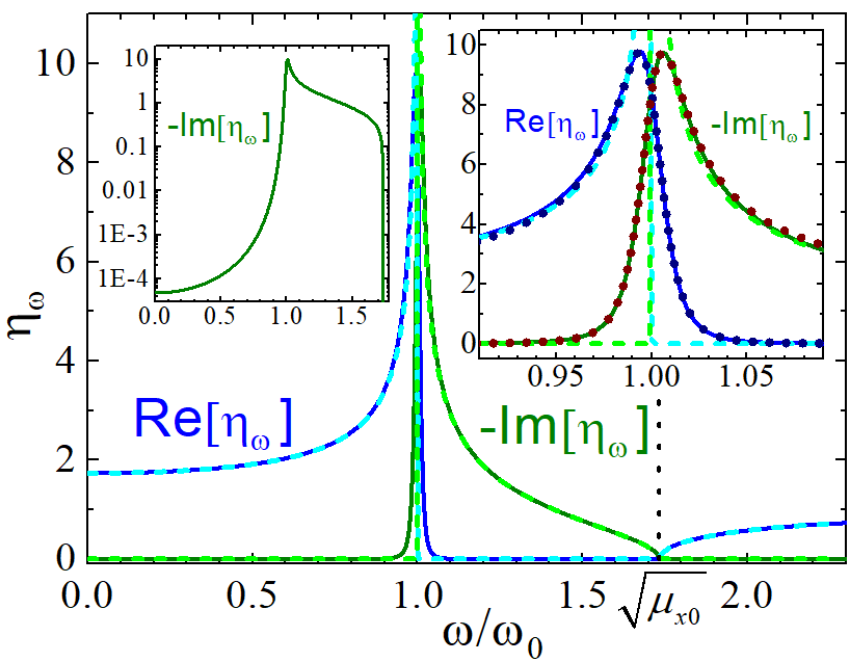

FIG. 5. The frequency dependences of the real and imaginary part of the parameter $\eta_{\omega}$, Eq. $68 \mathrm{~b}$, which determines the dynamic magnetic boundary condition, Eq. (68a). The dashed lines show the approximate result in Eq. (69) valid away from the frequency $\omega_{0}$. The right inset shows zoom in the region near the frequency $\omega_{0}$. The navy and wine dotted lines in this inset show the approximate scaling result in Eq. (71). The left inset shows the logarithmic plot of $-\operatorname{Im}\left[\eta_{\omega}\right]$ to illustrate that it remains finite down to zero frequency. The plots are made for $\zeta_{0}=0.02 \lambda$ and $\mu_{x 0}=3$.

In the range $\left|\omega^{2} / \omega_{0}^{2}-1\right| \gg \sqrt{\mu_{x 0}} \zeta_{0} / \lambda$ the parameters $\kappa_{\alpha}$ are given by Eqs. (64a) and 64b. In this case $\left|\kappa_{1}\right| \gg$ $\left|\kappa_{2}\right|, \lambda^{-1}$ and we obtain a simple approximate result

$$
\eta_{\omega} \approx \lambda \kappa_{2} \approx-i \sqrt{\frac{\mu_{x 0}-\omega^{2} / \omega_{0}^{2}}{\omega^{2} / \omega_{0}^{2}-1}} .
$$

Note that this result corresponds to the approximation of local magnetic response and it remains valid even in the regime where $\zeta_{0}\left|\kappa_{1}\right|>1$. In particular, it gives correctly the static-case result $\eta_{\omega=0}=\sqrt{\mu_{x 0}}$. On the other hand, at $\omega=\omega_{0}$, using Eq. 63), we obtain

$$
\eta_{\omega_{0}} \approx(1-i)\left(\mu_{x 0}-1\right)^{1 / 4} \sqrt{\lambda / \zeta_{0}} .
$$

In the range $\omega^{2} / \omega_{0}^{2}-1 \ll 2\left(\mu_{x 0}-1\right)$, we derive the following approximate scaling form,

$$
\begin{aligned}
\eta_{\omega} & \approx \sqrt{\lambda / \zeta_{0}}\left(\mu_{x 0}-1\right)^{1 / 4} v\left(\frac{\omega^{2} / \omega_{0}^{2}-1}{\zeta_{0} / \lambda \sqrt{\mu_{x 0}-1}}\right), \\
v(u) & =\frac{1}{1+i u}\left(\sqrt{\sqrt{\frac{u^{2}}{4}+1}+\frac{u}{2}}-i \sqrt{\sqrt{\frac{u^{2}}{4}+1}}-\frac{u}{2}\right) .
\end{aligned}
$$

The real and imaginary parts of the complex function $v(u)$ are connected by the relation $\operatorname{Re}[v(-u)]=$ $-\operatorname{Im}[v(u)]$. The real part reaches the maximum value equal to 1.162 at $u \approx-0.436$. The asymptotics of $v(u)$ in the range $u \gg 1$ is $v(u) \simeq-i / \sqrt{u}$ yielding $\eta_{\omega} \approx-i \sqrt{\mu_{x 0}-1} / \sqrt{\omega^{2} / \omega_{0}^{2}-1}$. This matches the result 


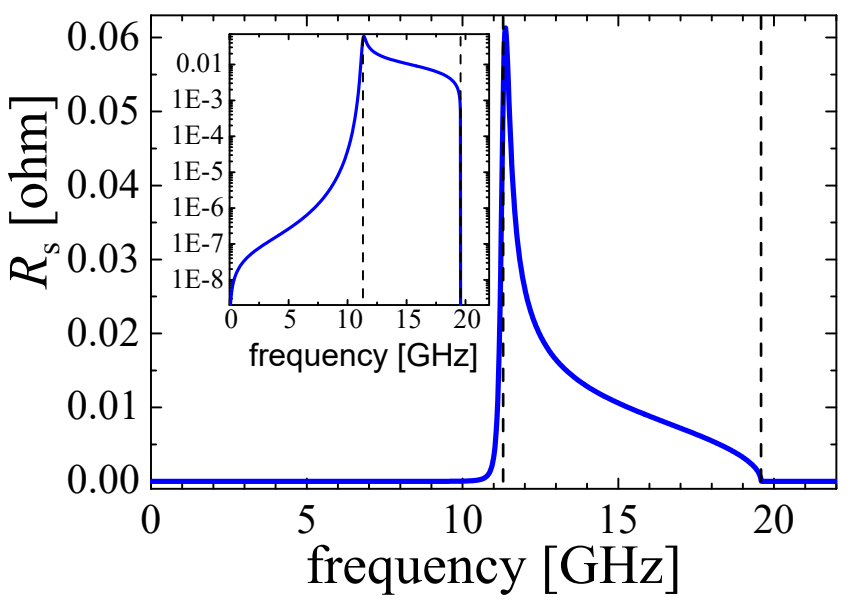

FIG. 6. The frequency dependence of the surface resistivity using the same parameters as in Figs. 2 and 5 corresponding to $\mathrm{RbEuFe}_{4} \mathrm{As}_{4}$. The inset shows the same plot in logarithmic scale for the better presentation of the low-frequency behavior. The vertical dashed lines show locations of the frequencies $f_{0}=\omega_{0} / 2 \pi$ and $\sqrt{\mu_{x 0}} f_{0}$.

in Eq. 690 in the range $\omega^{2} / \omega_{0}^{2}-1 \ll 1$. In the large negative region, $u<0,|u| \gg 1$, the imaginary part of $v(u)$ decays as $\operatorname{Im}[v(u)] \simeq-|u|^{-7 / 2}$.

Figure 5 shows plots of the real and imaginary part of the parameter $\eta_{\omega}$, Eq. 68b), computed using typical parameters $\zeta_{0}=0.02 \lambda$ and $\mu_{x 0}=3$. We also show in the figure the approximate result, Eq. (69), valid for frequencies not very close to $\omega_{0}$, and, in the upper right inset, the approximate scaling result in Eq. (71) describing behavior near $\omega_{0}$. The frequency dependence of $\eta_{\omega}$ can be summarized as follows. In the range $\omega<\omega_{0}$, the real part of $\eta_{\omega}$ is much larger than $-\operatorname{Im}\left(\eta_{\omega}\right)$. Both parts increase for $\omega \rightarrow \omega_{0}$ and become equal in absolute value at $\omega=\omega_{0}$. The real part reaches maximum $1.162 \sqrt{\lambda / \zeta_{0}}\left(\mu_{x 0}-1\right)^{1 / 4}$ slightly below $\omega_{0}$, at $\omega \approx \omega_{0}\left(1-0.218 \zeta_{0} / \lambda \sqrt{\mu_{x 0}-1}\right)$, while $-\operatorname{Im}\left(\eta_{\omega}\right)$ reaches the same maximum slightly above $\omega_{0}$, at $\omega \approx \omega_{0}\left(1+0.218 \zeta_{0} / \lambda \sqrt{\mu_{x 0}-1}\right)$. In the range $\omega_{0}<\omega<\sqrt{\mu_{x 0}} \omega_{0}$, the real part of $\eta_{\omega}$ is much smaller than $-\operatorname{Im}\left(\eta_{\omega}\right)$. Finally, in the region $\omega>\sqrt{\mu_{x 0}} \omega_{0}$ the imaginary part is zero, while the real part monotonically increases asymptotically approaching unity.

The parameter $\eta_{\omega}$ is directly connected with the conventional parameter characterizing the microwave response, surface impedance

$$
Z=\frac{E_{x}}{\int_{0}^{\infty} j_{x}(z) d z}=\frac{4 \pi}{\mathrm{c}} \frac{E_{x}}{H_{y}} .
$$

To establish this connection, we have to relate the tangential electric field with the normal gradient of the magnetic field. At small frequencies, we can use the London relation $\frac{4 \pi}{c} \frac{\partial j_{x}}{\partial t} \approx c \lambda^{-2} E_{x}$ neglecting a small contribution from the quasiparticle current and the Maxwell equation $-\nabla_{z} H_{y}=\frac{4 \pi}{\mathrm{c}} j_{x}$ omitting the displacement current. This

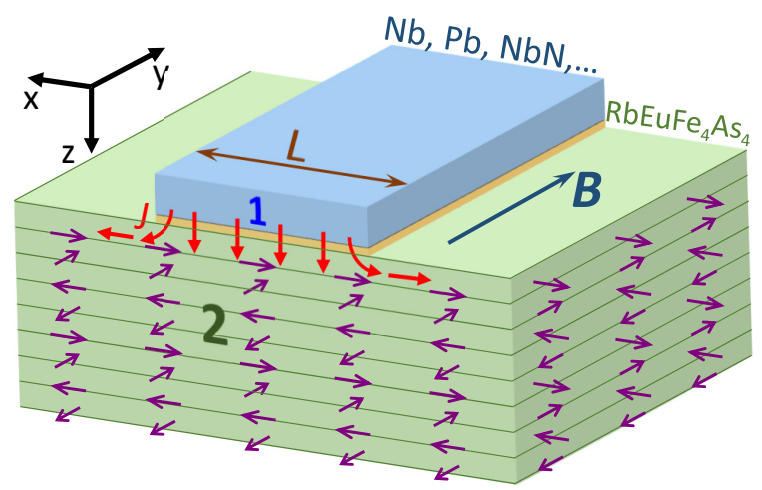

FIG. 7. Illustration of a planar tunneling contact between a conventional superconductor (1) and magnetic superconductor with helical magnetic structure (2). Purple arrows illustrate orientation of the magnetic moments.

gives $-\nabla_{z} H_{y}=\frac{\mathrm{c}}{i \omega \lambda^{2}} E_{x}$ and from Eq. 68a we obtain

$$
Z=4 \pi i \omega \eta_{\omega} \lambda / \mathrm{c}^{2}
$$

The real part of this equation, $R_{s}=\operatorname{Re}(Z)$, can be converted to the practical formula for surface resistivity $R_{s}[\mathrm{ohm}]=-8 \pi^{2} 10^{-4} \operatorname{Im}\left[\eta_{\omega}\right] f[\mathrm{GHz}] \lambda[\mu \mathrm{m}]$.

Figure 6 shows the frequency dependence of the surface resistivity using the same parameters as in Figs. 2 and 5 . We can see that the surface resistivity has a very distinct shape. It is very small at small frequencies $f<f_{0}=\omega_{0} / 2 \pi$ and starts to increase sharply when the frequency approaches $f_{0}$. After reaching a peak value $\sim 0.06 \mathrm{ohm}$ slightly above $f_{0}$, it slowly decreases within extended frequency range $f_{0}<f<\sqrt{\mu_{x 0}} f_{0}$, and abruptly vanishes at $\sqrt{\mu_{x 0}} f_{0}$.

\section{EXCITATION OF SPIN WAVES WITH AC JOSEPHSON EFFECT}

The presence of magnetic order inside superconducting material provides a unique possibility to generate and manipulate magnons using the AC Josephson effect. In this section, we consider the excitation of spin waves in a tunneling contact between a conventional superconductor marked by the index 1 and a superconductor with helical magnetic structure marked by the index 2 , as illustrated in Fig. 7. We assume that the system is uniform along the $y$ direction and the interlayer with thickness $\mathfrak{t}$ is insulating and nonmagnetic. The magnetic and conventional superconductors occupy the regions $z>0$ and $z<-\mathfrak{t}$, respectively.

\section{A. Dynamic equation for the Josephson phase}

We follow the standard derivation of the dynamic equation for the gauge-invariant phase difference between two 
superconductors $\theta=\phi_{2}-\phi_{1}-\frac{2 \pi t}{\Phi_{0}} A_{z}$ taking into account the dynamic magnetization response. The starting point of derivation is the $z$ component of the Maxwell equation,

$$
\nabla_{x} H_{y}=\frac{4 \pi}{\mathrm{c}} j_{z}+\frac{\varepsilon}{\mathrm{c}} \frac{\partial E_{z}}{\partial t}
$$

in which the total current density across the junction is composed of the superconducting and quasiparticle contributions, $j_{z}=j_{s, z}+j_{n, z}$, where the superconducting contribution is given by the DC Josephson relation,

$$
j_{s, z}=j_{J} \sin \theta,
$$

and the quasiparticle contribution is determined by tunneling conductivity $\sigma, j_{n, z}=\sigma E_{z}$. The electric field is related to the phase by the AC Josephson relation

$$
E_{z}=\frac{\Phi_{0}}{2 \pi c d} \frac{\partial \theta}{\partial t} .
$$

To relate $\nabla_{x} H_{y}$ in Eq. (74) with the phase gradient, we use the $x$ component of the Maxwell equations $-\nabla_{z} H_{y}=$ $\frac{4 \pi}{\mathrm{c}} j_{x}$ and the London relation for supercurrents along the junction $\frac{4 \pi}{\mathrm{c}} j_{x} \approx \lambda_{i}^{-2}\left(\frac{\Phi_{0}}{2 \pi} \nabla_{x} \phi-A_{x}\right)$. Here, we neglected the displacement current assuming small frequencies and quasiparticle current inside the superconductors. This leads to the relation between the in-plane phase gradient and magnetic fields

$$
\begin{aligned}
& \nabla_{x} \theta=\frac{8 \pi^{2}}{\mathrm{c} \Phi_{0}}\left(\lambda_{2}^{2} j_{x, 2}-\lambda_{1}^{2} j_{x, 1}\right)+\frac{2 \pi \mathfrak{t}}{\Phi_{0}} B_{y} \\
& =-\frac{2 \pi}{\Phi_{0}}\left(\lambda_{2}^{2} \nabla_{z} H_{y, 2}-\lambda_{1}^{2} \nabla_{z} H_{y, 1}\right)+\frac{2 \pi \mathfrak{t}}{\Phi_{0}} B_{y},
\end{aligned}
$$

where $j_{x, i}$ and $H_{y, i}$ are the current densities and the magnetic fields at the surfaces of two superconductors and $B_{y}$ is the magnetic induction inside the junction. We assume a nonmagnetic interlayer meaning that $B_{y}=H_{y}$. Also, for the nonmagnetic superconductor in the Meissner state at $z<-\mathfrak{t}$, we have $\nabla_{z} H_{y, 1}=B_{y} / \lambda_{1}$. To obtain the close system, we need the boundary condition connecting $\nabla_{z} H_{y, 2}$ with $H_{y}=B_{y}$ at the surface of the magnetic superconductor at $z=0$. Note that $H_{y}(z)$ is continuous, while $B_{y}(z)$ has a jump at $z=0$. Due to magnetization dynamics, this boundary condition is frequency dependent. At fixed frequency, such boundary condition has been derived in Sec. VII and is given by Eq. (68a), which in our case becomes $\nabla_{z} H_{y, 2}=-\eta_{\omega} B_{y} / \lambda_{2}$. The complex parameter $\eta_{\omega}$ is determined by the general result in Eq. 68b). In the approximation of local magnetic response valid for frequencies not too close to the bare uniform-mode frequency $\omega_{0}$, it has a much simpler approximate presentation in Eq. (69). Therefore, Eq. (77) at finite frequency becomes

$$
\nabla_{x} \theta=\frac{2 \pi\left(\mathfrak{t}+\lambda_{1}+\eta_{\omega} \lambda_{2}\right)}{\Phi_{0}} H_{y}
$$

Applying $\nabla_{x}$ to both sides, substituting $\nabla_{x} H_{y}$ from Eq. (74), and using the Josephson relations for current and electric field, Eqs. 75 and (76), we obtain the dynamic phase equation at finite frequency in the form

$$
\frac{1}{\mathfrak{t}+\lambda_{1}+\eta_{\omega} \lambda_{2}} \nabla_{x}^{2} \theta=\frac{8 \pi^{2}}{c \Phi_{0}} j_{J}[\sin \theta]_{\omega}-\frac{\varepsilon_{\omega} \omega^{2}}{\mathfrak{t} c^{2}} \theta
$$

where $\varepsilon_{\omega} \equiv \varepsilon-4 \pi i \sigma / \omega$ and $[\sin \theta]_{\omega}$ notates the time Fourier transform of $\sin [\theta(x, t)]$. The only difference from the standard phase-dynamics Sine-Gordon equation [66. 67] is the presence of the complex factor $\eta_{\omega}$ with complicated frequency dependence, see Fig. 5. In the static case, the phase equation is

$$
\frac{1}{\mathfrak{t}+\lambda_{1}+\sqrt{\mu_{x 0}} \lambda_{2}} \nabla_{x}^{2} \theta=\frac{8 \pi^{2}}{\mathrm{c} \Phi_{0}} j_{J} \sin \theta .
$$

Therefore, the effective junction interlayer width $\tilde{\mathfrak{t}}=\mathfrak{t}+$ $\lambda_{1}+\sqrt{\mu_{x 0}} \lambda_{2}$ is enlarged by the magnetic response. From the last equation, we can evaluate the static Josephson length

$$
\lambda_{J}=\left\{\frac{c \Phi_{0}}{\left[8 \pi^{2}\left(\mathfrak{t}+\lambda_{1}+\sqrt{\mu_{x 0}} \lambda_{2}\right) j_{J}\right]}\right\}^{1 / 2} .
$$

In the next subsection we consider the influence of magnetic response on the spectrum and damping of the electromagnetic wave propagating through the Josephson junction.

\section{B. Spectrum and damping of the Josephson plasma mode}

The superconductor-insulator-superconductor sandwich structure with sufficiently large width is a waveguide capable of supporting a traveling electromagnetic wave [67, 68, with the phase $\theta(x, t) \propto \exp \left[i\left(\omega_{w} t \pm k x\right)\right]$. Such a wave can be resonantly excited by the AC Josephson effect. For the fixed real wave vector $k$, Eq. 779 gives the following equation for the complex frequency $\omega_{w}(k)=\omega_{w, r}(k)+i \omega_{w, i}(k)$, with the real and imaginary part giving the wave spectrum and its damping, respectively,

$$
\omega_{w}^{2}-\frac{4 \pi \sigma}{\varepsilon} i \omega_{w}=\omega_{p}^{2}+\frac{\mathfrak{t}}{\mathfrak{t}+\lambda_{1}+\eta_{\omega} \lambda_{2}} \frac{\mathrm{c}^{2}}{\varepsilon} k^{2},
$$

where

$$
\omega_{p}=\sqrt{\frac{8 \pi^{2} c t}{\varepsilon \Phi_{0}} j_{J}}
$$

is the Josephson plasma frequency. Note that the magnetic response does not modify this parameter. It is convenient to rewrite Eq. 82 in the reduced form

$$
\frac{\omega_{w}^{2}}{\omega_{p}^{2}}-i \nu_{\sigma} \frac{\omega_{w}}{\omega_{p}}=1+\frac{\lambda_{1}+\sqrt{\mu_{x 0}} \lambda_{2}}{\lambda_{1}+\eta_{\omega} \lambda_{2}} \lambda_{J}^{2} k^{2}
$$




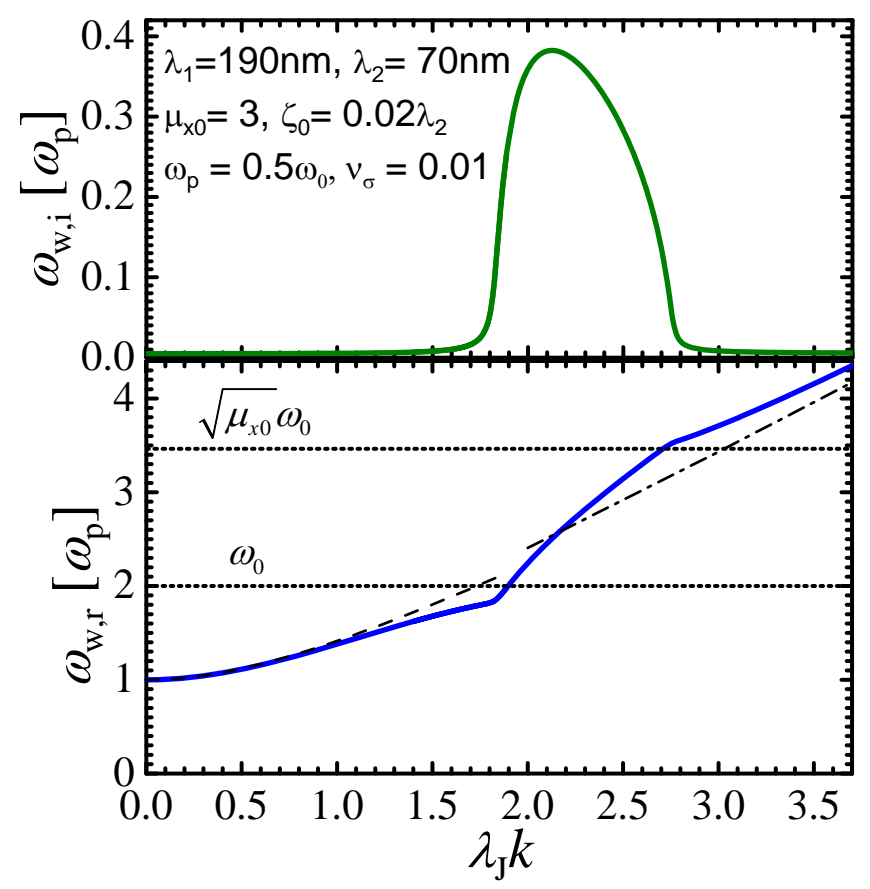

FIG. 8. Spectrum and damping of the electromagnetic wave inside a Josephson junction between conventional and helical magnetic superconductors, Eq. 84. In the lower plot, the dashed and dash-dotted lines show spectra corresponding to low-frequency and high-frequency limits, respectively.

with the static Josephson length $\lambda_{J}$, Eq. 81, and the dumping parameter

$$
\nu_{\sigma}=\frac{4 \pi \sigma}{\varepsilon \omega_{p}} .
$$

The parameter $\eta_{\omega}$ has the strongest feature around $\omega=$ $\omega_{0}$. Therefore, the spectrum of the Josephson plasmon is substantially affected only if $\omega_{p}<\omega_{0}$.

Figure 8 shows the spectrum and damping of the propagating wave computed from Eq. 84 for parameters corresponding to the contact between $\mathrm{NbN}$ and $\mathrm{RbEuFe}_{4} \mathrm{As}_{4}, \lambda_{1}=190 \mathrm{~nm}, \lambda_{2}=70 \mathrm{~nm}, \mu_{x 0}=3$, and $\varsigma_{0}=$ $0.02 \lambda_{2}$. We also assume $\omega_{p}=0.5 \omega_{0}$ and $\nu_{\sigma}=0.01$. One can distinguish three regions with qualitatively different behavior. In the low-frequency region $\omega_{w, r}(k)<\omega_{0}$, the spectrum is approximately $\omega_{w, r}(k) \simeq \sqrt{\omega_{p}^{2}+c_{s 0}^{2} k^{2}}$, where $c_{s 0}$ is the low-frequency Swihart velocity

$$
c_{s 0}=\lambda_{J} \omega_{p}=\sqrt{\frac{\mathfrak{t}}{\lambda_{1}+\sqrt{\mu_{x 0}} \lambda_{2}}} \frac{\mathrm{c}}{\sqrt{\varepsilon}} .
$$

In this region the spin waves give a small contribution to the mode damping. The intermediate region $\omega_{0}<\omega_{w, r}(k)<\sqrt{\mu_{x 0}} \omega_{0}$ is characterized by a sharp enhancement of the damping caused by excitation of the spin waves. Finally, in the high-frequency region $\omega_{w, r}(k)>\sqrt{\mu_{x 0}} \omega_{0}$ the damping caused by spin waves is absent and the spectrum approaches the high-frequency limit $\omega_{w, r}(k) \simeq \sqrt{\omega_{p}^{2}+c_{s 1}^{2} k^{2}} \simeq c_{s 1} k$, where $c_{s 1}$ is the high-frequency mode velocity,

$$
\begin{aligned}
c_{s 1} & =\sqrt{\frac{d}{\lambda_{1}+\lambda_{2}}} \frac{\mathrm{c}}{\sqrt{\varepsilon}} \\
& =\sqrt{\frac{\lambda_{1}+\sqrt{\mu_{x 0}} \lambda_{2}}{\lambda_{1}+\lambda_{2}}} c_{s 0} .
\end{aligned}
$$

In this limit the influence of magnetism is weak.

\section{Current-voltage characteristics and Fiske resonances in finite magnetic field}

Transport properties of a Josephson junction in the magnetic field directly probe its dynamic response [66, 67, 69, 70. In particular, one can directly excite collective modes in superconducting materials and the spectrum of these modes can be inferred from the dynamic features in the current-voltage characteristics [71. In this section, we evaluate the current-voltage characteristics for our system using the standard approach of the expansion with respect to the Josephson current assuming fixed voltage [72. Consider a junction in finite magnetic field $B_{y}$ and in the resistive state with finite voltage drop across the junction, $V=\mathfrak{t} E_{z}$. In this state, in the zeroth order with respect to the Josephson current, the phase has the shape of a traveling wave

$$
\theta_{0}(x, t)=k_{B} x+\omega t
$$

with the wave vector

$$
k_{B}=\frac{2 \pi}{\Phi_{0}}\left(\mathfrak{t}+\lambda_{1}+\sqrt{\mu_{x 0}} \lambda_{2}\right) B_{y},
$$

and the Josephson frequency

$$
\omega=\frac{2 \pi \mathrm{c}}{\Phi_{0}} V .
$$

Representing $\sin \theta_{0}(x, t)=\operatorname{Re}\left[-i \exp \left(i k_{B} x+i \omega t\right)\right]$, we obtain from Eq. (79p the equation for the first-order correction to the dynamic phase, $\tilde{\theta}(x, t)=\operatorname{Re}[\tilde{\theta}(x) \exp (i \omega t)]$, which we present as

$$
\nabla_{x}^{2} \tilde{\theta}+p_{\omega}^{2} \tilde{\theta}=-i r_{\omega} \lambda_{J}^{-2} \exp \left(i k_{B} x\right)
$$

with

$$
\begin{aligned}
r_{\omega} & \equiv \frac{\mathfrak{t}+\lambda_{1}+\eta_{\omega} \lambda_{2}}{\mathfrak{t}+\lambda_{1}+\sqrt{\mu_{x 0}} \lambda_{2}}, \\
p_{\omega}^{2} & \equiv \frac{\varepsilon_{\omega} \omega^{2}}{c^{2}} \frac{\mathfrak{t}+\lambda_{1}+\eta_{\omega} \lambda_{2}}{\mathfrak{t}} \\
& =\frac{\omega^{2}-(4 \pi \sigma / \varepsilon) i \omega}{c_{s 0}^{2}} r_{\omega},
\end{aligned}
$$

where $c_{s 0}$ is the low-frequency Swihart velocity, Eq. 86. 
We look for the solution of Eq. 91 in the form

$\tilde{\theta}(x)=\frac{r_{\omega} \lambda_{J}^{-2}}{k_{B}^{2}-p_{\omega}^{2}}\left[A_{c} \cos \left(p_{\omega} x\right)+A_{s} \sin \left(p_{\omega} x\right)+i \exp \left(i k_{B} x\right)\right]$

Assuming the nonradiative boundary conditions, $\nabla_{x} \tilde{\theta}=0$ for $x=0, L$, we find the coefficients $A_{c}$ and $A_{s}$,

$$
\begin{aligned}
A_{s} & =k_{B} / p_{\omega} \\
A_{c} \sin \left(p_{\omega} L\right) & =\frac{k_{B}}{p_{\omega}}\left[\cos \left(p_{\omega} L\right)-\exp \left(i k_{B} L\right)\right]
\end{aligned}
$$

and substitute them into Eq. (93). This yields the oscillating phase

$$
\tilde{\theta}(x)=\frac{r_{\omega} \lambda_{J}^{-2}}{k_{B}^{2}-p_{\omega}^{2}}\left[\frac{k_{B}}{p_{\omega}} \frac{\cos \left[p_{\omega}(L-x)\right]-\exp \left(i k_{B} L\right) \cos \left(p_{\omega} x\right)}{\sin \left(p_{\omega} L\right)}+i \exp \left(i k_{B} x\right)\right] .
$$

The average Josephson current density is given by

$$
\begin{aligned}
\delta j & =\frac{j_{J}}{L} \int_{0}^{L}\left\langle\sin \left(k_{B} x+\omega t+\operatorname{Re}[\tilde{\theta}(x) \exp (i \omega t)]\right)\right\rangle_{t} d x \\
& \approx \frac{j_{J}}{2 L} \int_{0}^{L} \operatorname{Re}\left[\tilde{\theta}(x) \exp \left(-i k_{B} x\right)\right] d x .
\end{aligned}
$$

Substituting $\tilde{\theta}(x)$ from Eq. 95, we obtain

$$
\begin{aligned}
& \delta j \approx \frac{j_{J} \lambda_{J}^{-2}}{2} \\
& \times \operatorname{Im}\left\{\left[1+\frac{\cos \left(p_{\omega} L\right)-\cos \left(k_{B} L\right)}{p_{\omega} L \sin \left(p_{\omega} L\right)} \frac{2 k_{B}^{2}}{p_{\omega}^{2}-k_{B}^{2}}\right] \frac{r_{\omega}}{p_{\omega}^{2}-k_{B}^{2}}\right\} .
\end{aligned}
$$

The key difference from the standard result 72 is the presence of the complex factor $\eta_{\omega}$ in the parameters $p_{\omega}$ and $r_{\omega}$ in Eqs. (92a) and (92b) from the magnetic boundary condition describing the excitation of spin waves in the magnetic superconductor. The location of the Fiske peaks corresponding to excitation of the standing electromagnetic waves inside the junction is determined by the condition $\operatorname{Re}\left[p_{\omega}\right] L=\pi n$. In the regions $\omega<\omega_{0}$ and $\omega>\sqrt{\mu_{x 0}} \omega_{0}$ where $\operatorname{Im}\left(\eta_{\omega}\right) \ll \operatorname{Re}\left(\eta_{\omega}\right)$, this condition gives the equation for the resonance frequencies

$$
\omega_{n}=\sqrt{\frac{\mathfrak{t}+\lambda_{1}+\sqrt{\mu_{x 0}} \lambda_{2}}{\mathfrak{t}+\lambda_{1}+\operatorname{Re}\left(\eta_{\omega}\right) \lambda_{2}}} \frac{\pi n \lambda_{J}}{L} \omega_{p},
$$

where $\omega_{p}$ is the Josephson plasma frequency, Eq. (83).

To facilitate numerical calculations, we rewrite Eq. (97) in the reduced form. We define the dimensionless size $\tilde{L}=L / \lambda_{J}$ and frequency $\tilde{\omega}=\omega / \omega_{p}$. We also introduce the reduced wave-vector parameters $\tilde{k}_{B}=\lambda_{J} k_{B}$ and

$$
\tilde{p}_{\omega}=\lambda_{J} p_{\omega}=\sqrt{\left(\tilde{\omega}^{2}-i \nu_{\sigma} \tilde{\omega}\right) r_{\omega}}
$$

where $\nu_{\sigma}$ is the dimensionless damping parameter,
Eq. 85. With these variables, we rewrite Eq. 97) as

$$
\frac{\delta j}{j_{J}}=\frac{1}{2} \operatorname{Im}\left\{\left[1+\frac{\cos \left(\tilde{p}_{\omega} \tilde{L}\right)-\cos \left(\tilde{k}_{B} \tilde{L}\right)}{\tilde{p}_{\omega} \tilde{L} \sin \left(\tilde{p}_{\omega} \tilde{L}\right)} \frac{2 \tilde{k}_{B}^{2}}{\tilde{p}_{\omega}^{2}-\tilde{k}_{B}^{2}}\right] \frac{r_{\omega}}{\tilde{p}_{\omega}^{2}-\tilde{k}_{B}^{2}}\right\} .
$$

The product $\tilde{k}_{B} \tilde{L}$ here may be related to the magnetic field as $\tilde{k}_{B} \tilde{L}=\pi B_{y} / B_{L}=2 \pi \Phi_{y} / \Phi_{0}$, where

$$
B_{L}=\frac{\Phi_{0}}{2 L\left(\mathfrak{t}+\lambda_{1}+\sqrt{\mu_{x 0}} \lambda_{2}\right)}
$$

is the size-dependent scale determining periodicity of magnetic oscillations of the Fiske resonances and $\Phi_{y}=$ $L\left(\mathfrak{t}+\lambda_{1}+\sqrt{\mu_{x 0}} \lambda_{2}\right) B_{y}$ is the magnetic flux through the junction. For frequency in Eq. (98), the strongest resonance is realized at $B=n B_{L}$. For other Fiske resonances, odd peaks with $n=2 m+1$ are maximal for $B_{y}=2 j B_{L}\left(\Phi_{y} / \Phi_{0}=j\right)$ while even peaks with $n=2 m$ are maximal for $B_{y}=(2 j+1) B_{L}\left(\Phi_{y} / \Phi_{0}=j+1 / 2\right)$ [66, 67, 72]. Adding the tunnel quasiparticle current, $j_{n}=\sigma E_{z}$, we obtain the total current in the reduced form

$$
\frac{j}{j_{J}}=\nu_{\sigma} \tilde{\omega}+\frac{\delta j}{j_{J}} .
$$

This equation together with Eq. (99) determines the current-voltage characteristic in the reduced form in the second order with respect to the Josephson current.

The shape of the current-voltage characteristic mostly depends on the relation between the Josephson plasma frequency $\omega_{p}$, the location of the first Fiske resonance $\omega_{1}=\pi c_{s 1} / L$, and the two typical spin-wave frequencies $\omega_{0}$ and $\sqrt{\mu_{x 0}} \omega_{0}$. As the resistive state is stable until the Josephson frequency exceeds the plasma frequency $\omega_{p}$, Eq. 83, spin waves can be excited only if $\omega_{p}$ is at least smaller than $\sqrt{\mu_{x 0}} \omega_{0}$. The clearest spin-wave features can be observed if $\omega_{p} \lesssim \omega_{0}$. In addition, the behavior is also very sensitive to the junction size $L$. For junctions narrower than the typical size $L_{c}=c_{s 1} \pi /\left(\sqrt{\mu_{x 0}} \omega_{0}\right)$, the whole spin-wave region $\omega_{0}<\omega<\sqrt{\mu_{x 0}} \omega_{0}$ is located below the Fiske resonances allowing for its clear resolution. For wider junctions the behavior is more complicated, because in this case the Fiske resonances are located both 


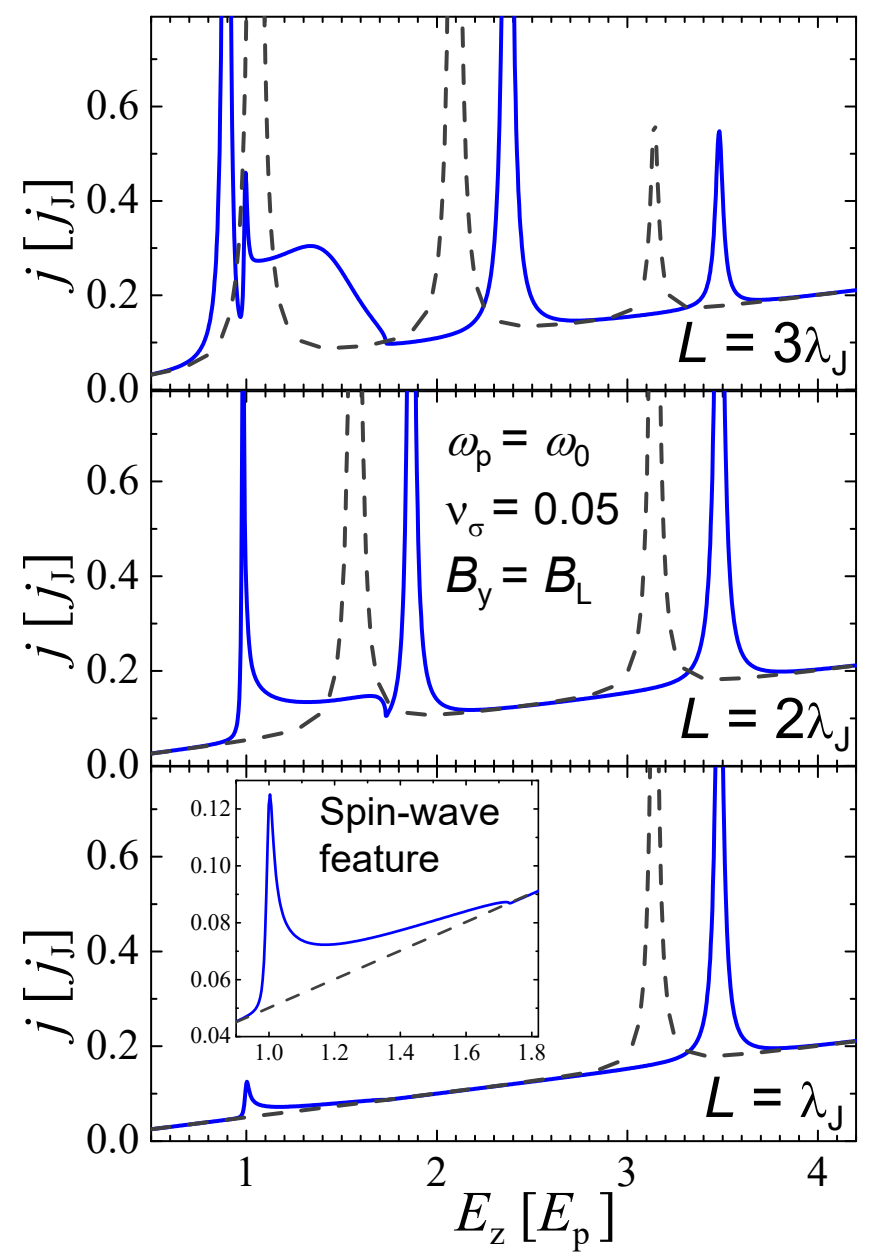

FIG. 9. Representative current-voltage characteristics for junctions with different lateral sizes $L$. The horizontal-axis scale $E_{p}$ is the electric field at which the Josephson frequency equals $\omega_{p}, E_{p}=\Phi_{0} \omega_{p} /(2 \pi c t)$. For comparison, the dashed lines show the current-voltage characteristics without dynamic magnetic response using static parameters $c_{s o}$ and $\lambda_{J}$. They display a usual sequence of the Fiske resonances. The inset in the bottom plot zooms into the spin-wave feature.

above and below the spin-wave region and some of them may fall inside this region. A very special situation is realized for the particular junction size $L_{\text {res }}$, at which the the first Fiske resonance is very close to $\omega_{0}$. To estimate this junction size, we substitute the maximum value of $\operatorname{Re}\left(\eta_{\omega}\right) \sim \sqrt{\lambda / \zeta_{0}}\left(\mu_{x 0}-1\right)^{1 / 4}$ to Eq. 98 at $n=1$ yielding

$$
L_{\mathrm{res}}=\pi \lambda_{J} \frac{\omega_{p}}{\omega_{0}} \sqrt{\frac{\mathfrak{t}+\lambda_{1}+\sqrt{\mu_{x 0}} \lambda_{2}}{\mathfrak{t}+\lambda_{1}+\left(\mu_{x 0}-1\right)^{1 / 4} \lambda_{2}^{3 / 2} / \sqrt{\zeta_{0}}}} .
$$

For this size, at the Josephson frequency slightly below $\omega_{0}$ the excited cavity mode generates the strongest spin wave inside the magnetic superconductor.

Figure 9 shows the representative current-voltage characteristics computed for the parameters $\omega_{p}=\omega_{0}, \nu_{\sigma}=$ 0.05 , three different sizes, $L / \lambda_{J}=1,2$, and 3 , and the magnetic field $B_{y}=B_{L}$. For reference, we also show by the dashed lines the current-voltage characteristics computed without dynamic magnetic response using static junction parameters. Note that (i) only the ascending left-side parts of the peaks are usually observed experimentally and (ii) the used linear approximation breaks in the middle of resonances meaning that the approximation overestimates the peak heights. We can see that there are substantial qualitative differences between the three shown cases. The junction size for the smallest junction is smaller than $L_{c}$ and therefore the spin-wave region is well below the Fiske resonances. The spin-wave feature has the same asymmetric shape as the surface resistivity in Fig. 6, it has a sharp peak when the Josephson frequency matches $\omega_{0}$ followed by an extended tail up to frequency $\sqrt{\mu_{x 0}} \omega_{0}$, see the inset in the bottom plot. The junction size $2 \lambda_{J}$ (middle plot) is very close to the resonance value $L_{\text {res }}$, Eq. 102, meaning that the spin-wave resonance at $\omega=\omega_{0}$ coincides with the first Fiske resonance leading to the very strong peak. A very peculiar feature of this case is that, due to strongly nonmonotonic behavior of $\operatorname{Re}\left(\eta_{\omega}\right)$ near the frequency $\omega_{0}$, the condition for the first resonance in Eq. (98) is satisfied at two frequencies, slightly below $\omega_{0}$ and slightly above $\sqrt{\mu_{x 0}} \omega_{0}$. Correspondingly, two strong peaks are realized at both frequencies. The largest size $3 \lambda_{J}$ exceeds both $L_{c}$ and $L_{\text {res }}$ (top plot). The first Fiske resonance in this case is located below $\omega_{0}$ and is slightly separated from the peak marking the onset of the spin-wave region. Correspondingly, the spin-wave region is located in between the first and second Fiske resonances. We also observe larger amplitude of the spin-wave feature in the region $\omega>\omega_{0}$. The reason is that the condition for the first resonance in Eq. (98) is also formally satisfied in the range $\omega_{0}<\omega<\sqrt{\mu_{x 0} \omega_{0}}$ where the absolute value of $\operatorname{Im}\left(\eta_{\omega}\right)$ is large marking very strong spin-wave damping of the resonance. As the resonance takes place in the overdamped region, it is seen as a shallow maximum.

The amplitudes of the Fiske resonances have oscillating dependence on the magnetic field [66, 67, 72]. Figure 10 shows the magnetic-field evolution of the current-voltage characteristics for the junction with $L=2 \lambda_{J}$. We see the familiar modulation of the resonances with magnetic field but with specific features. We see that the first two peaks have a similar dependence on the magnetic field, since they both represent the first Fiske resonance, while the third peak representing the second Fiske resonance is shifted by a half period. Note that the maximums of the first two peaks at $B=B_{L}$ and maximum of the third peak at $B=2 B_{L}$ are out of this general trend because they correspond to Eck resonance, $\omega=c_{s i} k_{B}$.

We demonstrated that the AC Josephson effect in a tunneling contact between conventional and helicalmagnetic superconductor can be utilized for the excitation of spin waves. Such excitation is most efficient when the Josephson frequency is in the range between the two typical spin-wave frequencies $\omega_{0}$ and $\sqrt{\mu_{x 0}} \omega_{0}$. In this range the current-voltage characteristic has a dis- 


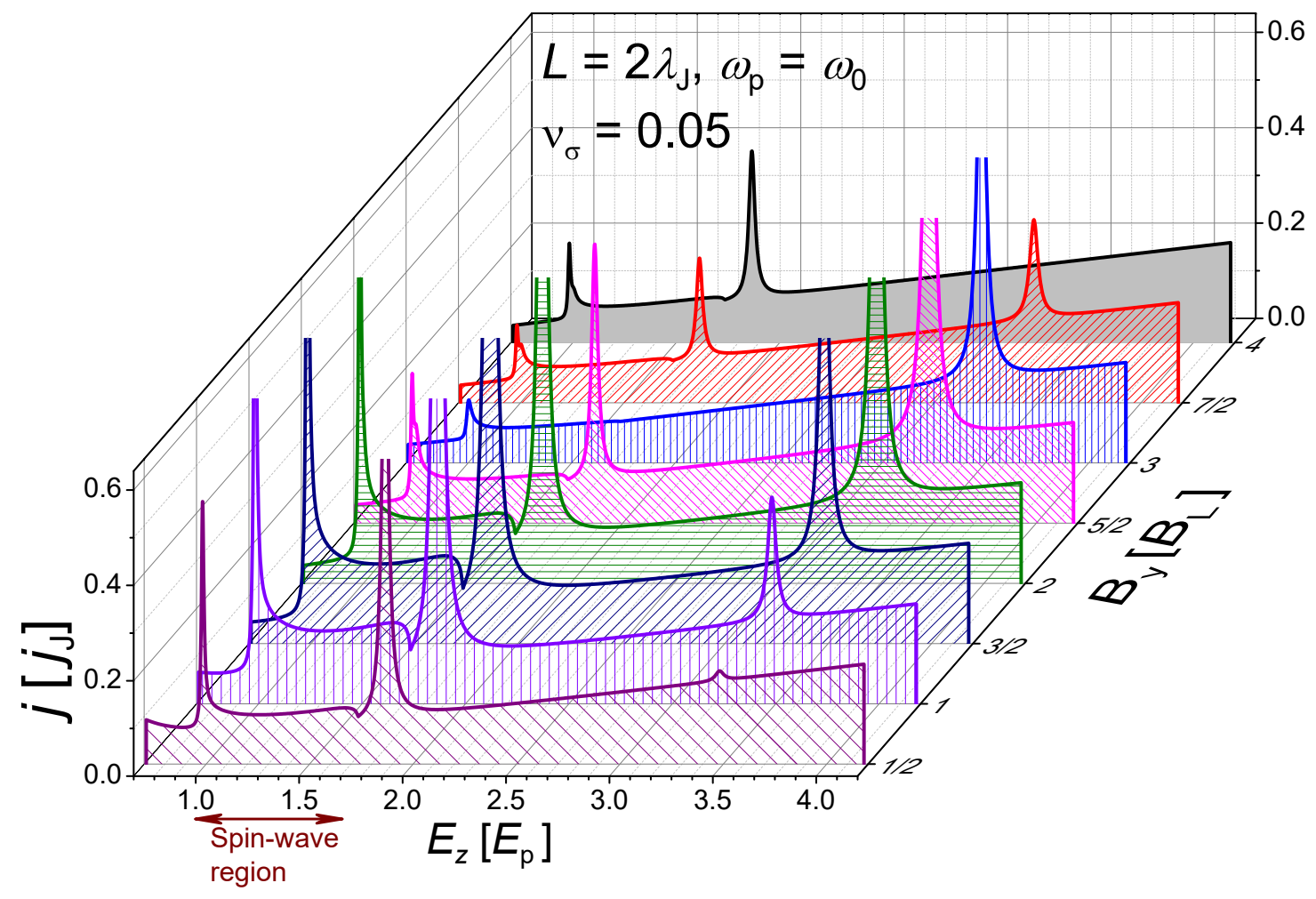

FIG. 10. The magnetic-field evolution of the current-voltage characteristics for junction with the parameters shown in the plot. The spin-wave feature is located in the region $1 \lesssim E_{z} / E_{p} \lesssim 1.7$. As the Fiske resonances, it is strongly modulated by the magnetic field.

tinct feature similar to one in the frequency dependence of the surface resistance. In addition, the spin-wave feature may strongly perturb the shape of Fiske resonances and the power of the excited spin wave may be enhanced when the Fiske resonance falls into the spin-wave region.

\section{SUMMARY AND DISCUSSION}

In summary, in this paper we consider spin waves and related observable effects in superconductors with helical magnetic order. Most computed specific results correspond to the structure realized in the iron pnictide $\mathrm{RbEuFe}_{4} \mathrm{As}_{4}$, in which the moments rotate $90^{\circ}$ from layer to layer, Fig. 1. The key feature of such materials is that the mode coupled with uniform field corresponds to the maximum frequency of the spin-wave spectrum with respect to the $c$-axis wave vector. The frequency of this mode is strongly enlarged by the long-range electromagnetic interactions between the oscillating magnetic moments and this enlargement rapidly vanishes when the $c$-axis wave-vector mismatch exceeds the inverse London penetration depth, see Fig. 22 For the parameters of $\mathrm{RbEuFe}_{4} \mathrm{As}_{4}$, we estimate the bare uniform-mode frequency $f_{0}$ as $\sim 11 \mathrm{GHz}$ and the renormalized one as $\sim 19$ $\mathrm{GHz}$, meaning that these frequencies are located within a convenient microwave range. We evaluate the frequency dependence of the surface resistance and find that it has a very distinct asymmetric spin-wave feature spreading between the bare and renormalized frequencies, see Fig. 6 .

We also investigate excitation of spin waves with the AC Josephson effect in a tunneling contact between helical-magnetic and conventional superconductors. For the most efficient excitation of spin waves, the Josephson plasma frequency has to be smaller than the bare uniform-mode frequency $\omega_{0}$. In addition, the features in the current-voltage characteristics are very sensitive to the junction size due to the interplay between the spin-wave excitation and Fiske resonances. The simplest behavior is realized in small-size junctions, when the renormalized frequency $\sqrt{\mu_{x 0}} \omega_{0}$ is below the lowest Fiske resonance. In this case, the whole spin-wave region is separated from the Fiske resonances and has a strongly asymmetric shape resembling the feature in the surface resistivity, see the inset in Fig. 9(bottom). In larger junctions, the Fiske resonances may fall inside the spin-wave region leading to more complicated behavior, see Fig. 9 (top and middle). The strongest excitation of the spin wave can be achieved in the situation when the Fiske resonance frequency is slightly below $\omega_{0}$ corresponding to the junction size in Eq. 102. As the Fiske resonances, the shape and amplitude of the spin-wave feature are modulated by magnetic field, see Fig. 10. We conclude that the AC Josephson effect provides a unique way to 
excite and manipulate spin waves in magnetic superconductors.

The author would like to acknowledge discussions with Ulrich Welp, Wai K. Kwok, Yi Li, and Valentine Novosad on possible experimental observations of the effects discussed in this paper. This work was supported by the US Department of Energy, Office of Science, Basic Energy Sciences, Materials Sciences and Engineering Division.
[1] L. Bulaevskii, A. Buzdin, M. Kulić, and S. Panjukov, Coexistence of superconductivity and magnetism theoretical predictions and experimental results, Adv. Phys. 34, 175 (1985).

[2] M. Kulić and A. I. Buzdin, Superconductivity, edited by K. H. Bennemann and J. B. Ketterson (Springer, Berlin, 2008) Chap. 4. Coexistence of Singlet Superconductivity andMagnetic Order in Bulk Magnetic Superconductors and SF Heterostructures, p. 163.

[3] C. T. Wolowiec, B. D. White, and M. B. Maple, Conventional magnetic superconductors, Physica C 514, 113 (2015)

[4] M. B. Maple and Ø. Fischer, eds., Superconductivity in Ternary Compounds II, Superconductivity and Magnetism (Springer, Berlin, 1982).

[5] P. W. Anderson and H. Suhl, Spin alignment in the superconducting state, Phys. Rev. 116, 898 (1959)

[6] L. N. Bulaevskii, A. I. Rusinov, and M. Kulić, Helical ordering of spins in a superconductor, J. Low Temp. Phys. 39, 255 (1980).

[7] M. Ishikawa and $\varnothing$. Fischer, Destruction of superconductivity by magnetic ordering in $\mathrm{Ho}_{1.2} \mathrm{Mo}_{6} \mathrm{~S}_{8}$, Solid State Commun. 23, 37 (1977)

[8] W. A. Fertig, D. C. Johnston, L. E. DeLong, R. W. McCallum, M. B. Maple, and B. T. Matthias, Destruction of superconductivity at the onset of long-range magnetic order in the compound $\mathrm{ErRh}_{4} \mathrm{~B}_{4}$, Phys. Rev. Lett. 38, 987 (1977)

[9] J. W. Lynn, G. Shirane, W. Thomlinson, and R. N. Shelton, Competition between ferromagnetism and superconductivity in $\mathrm{HoMo}_{6} \mathrm{~S}_{8}$, Phys. Rev. Lett. 46, 368 (1981)

[10] J. W. Lynn, G. Shirane, W. Thomlinson, R. N. Shelton, and D. E. Moncton, Magnetic properties of the reentrant ferromagnetic superconductor $\mathrm{HoMo}_{6} \mathrm{~S}_{8}$, Phys. Rev. B 24, 3817 (1981).

[11] D. E. Moncton, D. B. McWhan, P. H. Schmidt, G. Shirane, W. Thomlinson, M. B. Maple, H. B. MacKay, L. D. Woolf, Z. Fisk, and D. C. Johnston, Oscillatory magnetic fluctuations near the superconductor-toferromagnet transition in $\operatorname{errh}_{4} \mathrm{~b}_{4}$, Phys. Rev. Lett. 45, 2060 (1980)

[12] K.-H. Müller and V. N. Narozhnyi, Interaction of superconductivity and magnetism in borocarbide superconductors, Rep. Prog. Phys. 64, 943 (2001).

[13] L. C. Gupta, Superconductivity and magnetism and their interplay in quaternary borocarbides $\mathrm{RNi}_{2} \mathrm{~B}_{2} \mathrm{C}$, $\mathrm{Adv}$. Phys. 55, 691 (2006)

[14] C. Mazumdar and R. Nagarajan, Quaternary borocarbides: Relatively high $\mathrm{T}_{c}$ intermetallic superconductors and magnetic superconductors, Physica C 514, 173 (2015)

[15] D. Aoki and J. Flouquet, Ferromagnetism and superconductivity in uranium compounds, J. Phys. Soc. Jpn. 81, $011003(2012)$

[16] D. Aoki, K. Ishida, and J. Flouquet, Review of U- based ferromagnetic superconductors: Comparison between $\mathrm{UGe}_{2}$, URhGe, and UCoGe, J. Phys. Soc. Jpn. 88, 022001 (2019).

[17] A. D. Huxley, Ferromagnetic superconductors, Physica C 514, $368(2015)$

[18] S. Ran, C. Eckberg, Q.-P. Ding, Y. Furukawa, T. Metz, S. R. Saha, I.-L. Liu, M. Zic, H. Kim, J. Paglione, and N. P. Butch, Nearly ferromagnetic spin-triplet superconductivity, Science 365, 684 (2019).

[19] S. Zapf and M. Dressel, Europium-based iron pnictides: a unique laboratory for magnetism, superconductivity and structural effects, Rep. Prog. Phys. 80, 016501 (2017).

[20] Z. Ren, Z. Zhu, S. Jiang, X. Xu, Q. Tao, C. Wang, C. Feng, G. Cao, and Z. Xu, Antiferromagnetic transition in $\mathrm{EuFe}_{2} \mathrm{As}_{2}$ : A possible parent compound for superconductors, Phys. Rev. B 78, 052501 (2008)

[21] H. S. Jeevan, Z. Hossain, D. Kasinathan, H. Rosner, C. Geibel, and P. Gegenwart, Electrical resistivity and specific heat of single-crystalline $\mathrm{EuFe}_{2} \mathrm{As}_{2}$ : A magnetic homologue of $\mathrm{SrFe}_{2} \mathrm{As}_{2}$, Phys. Rev. B 78, 052502 (2008).

[22] Y. Xiao, Y. Su, W. Schmidt, K. Schmalzl, C. M. N. Kumar, S. Price, T. Chatterji, R. Mittal, L. J. Chang, S. Nandi, N. Kumar, S. K. Dhar, A. Thamizhavel, and T. Brueckel, Field-induced spin reorientation and giant spin-lattice coupling in $\mathrm{EuFe}_{2} \mathrm{As}_{2}$, Phys. Rev. B 81, 220406(R) (2010)

[23] C. F. Miclea, M. Nicklas, H. S. Jeevan, D. Kasinathan, Z. Hossain, H. Rosner, P. Gegenwart, C. Geibel, and F. Steglich, Evidence for a reentrant superconducting state in $\mathrm{EuFe}_{2} \mathrm{As}_{2}$ under pressure, Phys. Rev. B 79, 212509 (2009); T. Terashima, M. Kimata, H. Satsukawa, A. Harada, K. Hazama, S. Uji, H. S. Suzuki, T. Matsumoto, and K. Murata, $\mathrm{EuFe}_{2} \mathrm{As}_{2}$ under high pressure: An antiferromagnetic bulk superconductor, J. Phys. Soc. Jpn. 78, 083701 (2009)

[24] Z. Ren, Q. Tao, S. Jiang, C. Feng, C. Wang, J. Dai, G. Cao, and Z. Xu, Superconductivity induced by phosphorus doping and its coexistence with ferromagnetism in $\mathrm{EuFe}_{2}\left(\mathrm{As}_{0.7} \mathrm{P}_{0.3}\right)_{2}$, Phys. Rev. Lett. 102, 137002 (2009)

[25] H. S. Jeevan, D. Kasinathan, H. Rosner, and P. Gegenwart, Interplay of antiferromagnetism, ferromagnetism, and superconductivity in $\mathrm{EuFe}_{2}\left(\mathrm{As}_{1-x} \mathrm{P}_{x}\right)_{2}$ single crystals, Phys. Rev. B 83, 054511 (2011).

[26] G. Cao, S. Xu, Z. Ren, S. Jiang, C. Feng, and $\mathrm{Z}$. Xu, Superconductivity and ferromagnetism in $\mathrm{EuFe}_{2}\left(\mathrm{As}_{1-x} \mathrm{P}_{x}\right)_{2}$, Journal of Physics: Condensed Matter 23, 464204 (2011).

[27] Y. Tokiwa, S.-H. Hübner, O. Beck, H. S. Jeevan, and $\mathrm{P}$. Gegenwart, Unique phase diagram with narrow superconducting dome in $\mathrm{EuFe}_{2}\left(\mathrm{As}_{1-x} \mathrm{P}_{x}\right)_{2}$ due to $\mathrm{Eu}^{2+}$ local magnetic moments, Phys. Rev. B 86, 220505(R) (2012)

[28] S. Zapf, H. S. Jeevan, T. Ivek, F. Pfister, F. Klingert, S. Jiang, D. Wu, P. Gegenwart, R. K. Kremer, and M. Dressel, $\operatorname{EuFe}_{2}\left(\mathrm{As}_{1-x} \mathrm{P}_{x}\right)_{2}$ : Reentrant spin glass and superconductivity, Phys. Rev. Lett. 110, 237002 (2013) 
[29] S. Nandi, W. T. Jin, Y. Xiao, Y. Su, S. Price, D. K. Shukla, J. Strempfer, H. S. Jeevan, P. Gegenwart, and T. Brückel, Coexistence of superconductivity and ferromagnetism in P-doped $\mathrm{EuFe}_{2} \mathrm{As}_{2}$, Phys. Rev. B 89, $014512(2014)$

[30] W.-H. Jiao, Q. Tao, J.-K. Bao, Y.-L. Sun, C.-M. Feng, Z.A. Xu, I. Nowik, I. Felner, and G.-H. Cao, Anisotropic superconductivity in $\mathrm{Eu}\left(\mathrm{Fe}_{0.75} \mathrm{Ru}_{0.25}\right)_{2} \mathrm{As}_{2}$ ferromagnetic superconductor, Europhys Lett 95, 67007 (2011).

[31] S. Jiang, H. Xing, G. Xuan, Z. Ren, C. Wang, Z.-a. $\mathrm{Xu}$, and G. Cao, Superconductivity and local-moment magnetism in $\mathrm{Eu}\left(\mathrm{Fe}_{0.89} \mathrm{Co}_{0.11}\right)_{2} \mathrm{As}_{2}$, Phys. Rev. B 80, 184514 (2009) Y. He, T. Wu, G. Wu, Q. J. Zheng, Y. Z. Liu, H. Chen, J. J. Ying, R. H. Liu, X. F. Wang, Y. L. Xie, Y. J. Yan, J. K. Dong, S. Y. Li, and X. H. Chen, Evidence for competing magnetic and superconducting phases in superconducting $\mathrm{Eu}_{1-x} \mathrm{Sr}_{x} \mathrm{Fe}_{2-y} \mathrm{Co}_{y} \mathrm{As}_{2}$ single crystals, J. Physics: Condens. Matter 22, 235701 (2010). Z. Guguchia, S. Bosma, S. Weyeneth, A. Shengelaya, R. Puzniak, Z. Bukowski, J. Karpinski, and H. Keller, Anisotropic magnetic order of the eu sublattice in single crystals of $\mathrm{EuFe}_{2-x} \mathrm{Co}_{x} \mathrm{As}_{2}(x=0,0.2)$ studied by means of magnetization and magnetic torque, Phys. Rev. B 84, 144506 (2011) W. T. Jin, S. Nandi, Y. Xiao, Y. Su, O. Zaharko, Z. Guguchia, Z. Bukowski, S. Price, W. H. Jiao, G. H. Cao, and T. Brückel, Magnetic structure of superconducting $\mathrm{Eu}\left(\mathrm{Fe}_{0.82} \mathrm{Co}_{0.18}\right)_{2} \mathrm{As}_{2}$ as revealed by single-crystal neutron diffraction, Phys. Rev. B 88, 214516 (2013)

[32] U. B. Paramanik, P. L. Paulose, S. Ramakrishnan, A. K. Nigam, C. Geibel, and Z. Hossain, Magnetic and superconducting properties of Ir-doped $\mathrm{EuFe}_{2} \mathrm{As}_{2}$, Supercond. Sci. Technol. 27, 075012 (2014)

[33] H. S. Jeevan, Z. Hossain, D. Kasinathan, H. Rosner, C. Geibel, and P. Gegenwart, High-temperature superconductivity in $\mathrm{Eu}_{0.5} \mathrm{~K}_{0.5} \mathrm{Fe}_{2} \mathrm{As}_{2}$, Phys. Rev. B 78, 092406 (2008)

[34] Y. Qi, Z. Gao, L. Wang, D. Wang, X. Zhang, and Y. Ma, Superconductivity at $34.7 \mathrm{~K}$ in the iron arsenide $\mathrm{Eu}_{0.7} \mathrm{Na}_{0.3} \mathrm{Fe}_{2} \mathrm{As}_{2}$, New J. Phys. 10, 123003 (2008).

[35] I. S. Veshchunov, L. Y. Vinnikov, V. S. Stolyarov, N. Zhou, Z. X. Shi, X. F. Xu, S. Y. Grebenchuk, D. S. Baranov, I. A. Golovchanskiy, S. Pyon, Y. Sun, W. Jiao, G. Cao, T. Tamegai, and A. A. Golubov, Visualization of the magnetic flux structure in phosphorus-doped $\mathrm{EuFe}_{2} \mathrm{As}_{2}$ single crystals, JETP Letters 105, 98 (2017)

[36] V. S. Stolyarov, I. S. Veshchunov, S. Y. Grebenchuk, D. S. Baranov, I. A. Golovchanskiy, A. G. Shishkin, N. Zhou, Z. Shi, X. Xu, S. Pyon, Y. Sun, W. Jiao, G.-H. Cao, L. Y. Vinnikov, A. A. Golubov, T. Tamegai, A. I. Buzdin, and D. Roditchev, Domain Meissner state and spontaneous vortex-antivortex generation in the ferromagnetic superconductor $\mathrm{EuFe}_{2}\left(\mathrm{As}_{0.79} \mathrm{P}_{0.21}\right)_{2}$, Sci. Adv. 4, eaat1061 (2018)

[37] Z. Devizorova, S. Mironov, and A. Buzdin, Theory of magnetic domain phases in ferromagnetic superconductors, Phys. Rev. Lett. 122, 117002 (2019).

[38] Y. Liu, Y.-B. Liu, Q. Chen, Z.-T. Tang, W.-H. Jiao, Q. Tao, Z.-A. Xu, and G.-H. Cao, A new ferromagnetic superconductor: $\mathrm{CsEuFe}_{4} \mathrm{As}_{4}$, Science Bulletin 61, 1213 (2016)

[39] K. Kawashima, T. Kinjo, T. Nishio, S. Ishida, H. Fujihisa, Y. Gotoh, K. Kihou, H. Eisaki, Y. Yoshida, and A. Iyo, Superconductivity in Fe-based compound
$\mathrm{EuAFe}_{4} \mathrm{As}_{4}(\mathrm{~A}=\mathrm{Rb}$ and Cs$)$, J. Phys. Soc. Jpn. 85, 064710 (2016)

[40] J.-K. Bao, K. Willa, M. P. Smylie, H. Chen, U. Welp, D. Y. Chung, and M. G. Kanatzidis, Single crystal growth and study of the ferromagnetic superconductor $\mathrm{RbEuFe}_{4} \mathrm{As}_{4}$, Crystal Growth \& Design 18, 3517 (2018)

[41] M. P. Smylie, K. Willa, J.-K. Bao, K. Ryan, Z. Islam, H. Claus, Y. Simsek, Z. Diao, A. Rydh, A. E. Koshelev, W.-K. Kwok, D. Y. Chung, M. G. Kanatzidis, and U. Welp, Anisotropic superconductivity and magnetism in single-crystal $\mathrm{RbEuFe}_{4} \mathrm{As}_{4}$, Phys. Rev. B 98, 104503 (2018)

[42] V. S. Stolyarov, A. Casano, M. A. Belyanchikov, A. S. Astrakhantseva, S. Y. Grebenchuk, D. S. Baranov, I. A. Golovchanskiy, I. Voloshenko, E. S. Zhukova, B. P. Gorshunov, A. V. Muratov, V. V. Dremov, L. Y. Vinnikov, D. Roditchev, Y. Liu, G.-H. Cao, M. Dressel, and E. Uykur, Unique interplay between superconducting and ferromagnetic orders in $\mathrm{EuRbFe}_{4} \mathrm{As}_{4}$, Phys. Rev. B 98, 140506(R) (2018)

[43] Y. Liu, Y.-B. Liu, Z.-T. Tang, H. Jiang, Z.-C. Wang, A. Ablimit, W.-H. Jiao, Q. Tao, C.-M. Feng, Z.-A. Xu, and G.-H. Cao, Superconductivity and ferromagnetism in hole-doped $\mathrm{RbEuFe}_{4} \mathrm{As}_{4}$, Phys. Rev. B 93, 214503 (2016)

[44] K. Willa, R. Willa, J.-K. Bao, A. E. Koshelev, D. Y. Chung, M. G. Kanatzidis, W.-K. Kwok, and U. Welp, Strongly fluctuating moments in the high-temperature magnetic superconductor $\mathrm{RbEuFe}_{4} \mathrm{As}_{4}$, Phys. Rev. B 99, 180502(R) (2019)

[45] M. Hemmida, N. Winterhalter-Stocker, D. Ehlers, H. A. K. von Nidda, M. Yao, J. Bannies, E. D. L. Rienks, R. Kurleto, C. Felser, B. Büchner, J. Fink, S. Gorol, T. Förster, S. Arsenijevic, V. Fritsch, and P. Gegenwart, Topological magnetic order and superconductivity in $\mathrm{EuRbFe}_{4} \mathrm{As}_{4}$ (2020), arXiv:2010.02110 [cond-mat.suprcon]

[46] D. E. Jackson, D. VanGennep, W. Bi, D. Zhang, P. Materne, Y. Liu, G.-H. Cao, S. T. Weir, Y. K. Vohra, and J. J. Hamlin, Superconducting and magnetic phase diagram of $\mathrm{RbEuFe}_{4} \mathrm{As}_{4}$ and $\mathrm{CsEuFe}_{4} \mathrm{As}_{4}$ at high pressure, Phys. Rev. B 98, 014518 (2018).

[47] L. Xiang, S. L. Bud'ko, J.-K. Bao, D. Y. Chung, M. G. Kanatzidis, and P. C. Canfield, Pressure-temperature phase diagram of the EuRbFe $\mathrm{As}_{4}$ superconductor, Phys. Rev. B 99, 144509 (2019).

[48] K. Iida, Y. Nagai, S. Ishida, M. Ishikado, N. Murai, A. D. Christianson, H. Yoshida, Y. Inamura, H. Nakamura, A. Nakao, K. Munakata, D. Kagerbauer, M. Eisterer, K. Kawashima, Y. Yoshida, H. Eisaki, and A. Iyo, Coexisting spin resonance and long-range magnetic order of $\mathrm{Eu}$ in $\mathrm{EuRbFe}_{4} \mathrm{As}_{4}$, Phys. Rev. B 100, 014506 (2019).

[49] Z. Islam, O. Chmaissem, A. E. Koshelev, J.-W. Kim, H. Cao, A. Rydh, M. P. Smylie, K. Willa, J. Bao, D. Y. Chung, M. Kanatzidis, W.-K. Kwok, S. Rosenkranz, and U. Welp, unpublished (2020).

[50] J. Van Kranendonk and J. H. Van Vleck, Spin waves, Rev. Mod. Phys. 30, 1 (1958).

[51] A. I. Akhiezer, V. G. Baryakhtar, and S. V. Peletminskii, Spin waves, North-Holland series in low temperature physics (North-Holland, Amsterdam, 1968).

[52] A. Prabhakar and D. D. Stancil, Spin waves: Theory and applications (Springer, Boston, 2009).

[53] S. A. Wolf, D. D. Awschalom, R. A. Buhrman, J. M. 
Daughton, S. von Molnár, M. L. Roukes, A. Y. Chtchelkanova, and D. M. Treger, Spintronics: A spinbased electronics vision for the future, Science 294, 1488 (2001)

[54] I. Zutić, J. Fabian, and S. Das Sarma, Spintronics: Fundamentals and applications, Rev. Mod. Phys. 76, 323 (2004)

[55] S. Neusser and D. Grundler, Magnonics: Spin waves on the nanoscale, Advanced Materials 21, 2927 (2009)

[56] V. V. Kruglyak, S. O. Demokritov, and D. Grundler, Magnonics, Journal of Physics D: Applied Physics 43, 264001 (2010)

[57] A. V. Chumak, V. Vasyuchka, A. Serga, and B. Hillebrands, Magnon spintronics, Nature Physics 11, 453 (2015)

[58] V. Braude and E. B. Sonin, Excitation of spin waves in superconducting ferromagnets, Phys. Rev. Lett. 93, 117001 (2004)

[59] V. Braude, Microwave response and spin waves in superconducting ferromagnets, Phys. Rev. B 74, 054515 (2006)

[60] A. I. Buzdin, Spin-wave spectrum of antiferromagnetic superconductors, JETP Lett. 40, 956 (1984), [Pis'ma Zh. Eksp. Teor. Fiz., 40193 (1984)].

[61] A. F. Volkov and K. B. Efetov, Hybridization of spin and plasma waves in josephson tunnel junctions containing a ferromagnetic layer, Phys. Rev. Lett. 103, 037003 (2009).

[62] S. Mai, E. Kandelaki, A. F. Volkov, and K. B. Efetov, Interaction of josephson and magnetic oscillations in josephson tunnel junctions with a ferromagnetic layer, Phys. Rev. B 84, 144519 (2011)

[63] A. E. Koshelev, Helical structures in layered magnetic su- perconductors due to indirect exchange interactions mediated by interlayer tunneling, Phys. Rev. B 100, 224503 (2019)

[64] T. Nagamiya, Helical spin ordering-1 Theory of helical spin configurations, in Solid State Physics, Vol. 20, edited by F. Seitz, D. Turnbull, and H. Ehrenreich (Academic Press, 1968) pp. 305-411.

[65] D. C. Johnston, Unified molecular field theory for collinear and noncollinear Heisenberg antiferromagnets, Phys. Rev. B 91, 064427 (2015).

[66] I. Kulik and I. Yanson, Josephson Effect in Superconducting Tunneling Structures (Israel Program for Scientific Translations, Jerusalem, 1972).

[67] A. Barone and G. Paterno, Physics and Applications of The Josephson Effect (Wiley, New York, 1982).

[68] J. C. Swihart, Field solution for a thin-film superconducting strip transmission line, J. Appl. Phys. 32, 461 (1961).

[69] M. Cirillo, N. Grønbech-Jensen, M. R. Samuelsen, M. Salerno, and G. V. Rinati, Fiske modes and Eck steps in long Josephson junctions: Theory and experiments, Phys. Rev. B 58, 12377 (1998)

[70] J. R. Kirtley, Magnetic field effects in Josephson junctions, in Fundamentals and Frontiers of the Josephson Effect, edited by F. Tafuri (Springer International Publishing, Cham, 2019) pp. 209-233.

[71] R. V. Carlson and A. M. Goldman, Dynamics of the order parameter of superconducting aluminum films, J. Low Temp. Phys. 25, 67 (1976)

[72] I. Kulik, Theory of "steps" of voltage-current characteristics of the Josephson tunnel current, JETP Lett. 2, 84 (1965), [Zh. Eksper. Teor. Pis. Red. 2, 134(1965)]. 